\title{
Numerical modeling of lander interaction with a low-gravity asteroid regolith surface
}

\section{Application to MASCOT on board Hayabusa2}

\author{
Florian Thuillet ${ }^{1}$, Patrick Michel ${ }^{1}$, Clara Maurel $^{2}$, Ronald-Louis Ballouz ${ }^{3,4}$, Yun Zhang ${ }^{3,5}$, Derek C. Richardson ${ }^{3}$, \\ Jens Biele ${ }^{6}$, Eri Tatsumi ${ }^{7}$, and Seiji Sugita ${ }^{7,8}$ \\ ${ }^{1}$ Lagrange Laboratory, Université Côte d'Azur, Observatoire de la Côte d'Azur, CNRS, CS 34229, 06304 Nice Cedex 4, France \\ e-mail: fthuille@oca.eu \\ ${ }^{2}$ Department of Earth and Planetary Sciences, Massachusetts Institute of Technology, 77 Massachusetts Avenue, Cambridge, \\ MA 02139, USA \\ ${ }^{3}$ Department of Astronomy, University of Maryland, College Park, MD 20742, USA \\ ${ }^{4}$ Institute for Space and Astronautical Studies, Japanese Aerospace eXploration Agency, Sagamihara, Kanagawa Prefecture \\ 252-5210, Japan \\ ${ }^{5}$ School of Aerospace Engineering, Tsinghua University, Beijing 100084, PR China \\ ${ }^{6}$ DLR German Aerospace Center, Micro-Gravity User Support Center, 51147 Cologne, Germany \\ ${ }^{7}$ Department of Earth and Planetary Science, University of Tokyo, 7-3-1 Hongo, Bunkyo-ku, Tokyo 113-0033, Japan \\ ${ }^{8}$ Research Center for the Early Universe, University of Tokyo, 7-3-1 Hongo, Bunkyo-ku, Tokyo 113-0033, Japan
}

Received 6 February 2018 / Accepted 6 April 2018

\begin{abstract}
Context. Landing on the surface of small bodies is particularly challenging, as the physical properties of the surface material are not well known and the mechanical response of this material in a low-gravity environment is not well understood.

Aims. In order to improve our understanding of low-speed impact processes on granular media and their outcome in low-gravity environments, we consider the landing of the package MASCOT, to be released by the JAXA asteroid sample return mission Hayabusa2 on (162173) Ryugu in October 2018. Beyond addressing the theoretical aspects of the mechanical response of granular media in low gravity, this study also supports both engineering and scientific teams of Hayabusa2 in the search for the lander and in the determination of Ryugu's surface properties.

Methods. A campaign of hundreds of numerical simulations using the soft-sphere discrete element method implemented in the $\mathrm{N}$-body code pkdgrav were performed to study the interaction between the lander and the low-gravity surface of the asteroid made of a granular medium representing the regolith. Assuming a broad range of regolith properties, and the lander's trajectory and motion, we analyzed the outcomes of the landing (distance traveled by the lander, penetration depth, and shape of the traces left in the regolith surface) to determine the influence of the many parameters defining the properties of MASCOT and of the grains, and the ingoing motion of the lander.

Results. We identify well-marked trends for the fate of the lander and the traces left in the granular material. Distances traveled by the lander are greater and penetrations are shallower for gravel-like media than for less frictional material. A similar trend is found for grazing impacts as opposed to vertical ones. Different regolith properties also generate different traces on the ground after the impact.
\end{abstract}

Key words. minor planets, asteroids: individual: (162173) Ryugu - methods: numerical

\section{Introduction}

The dynamics of granular material, and the material's response to external actions, is an active domain of research with various industrial and scientific applications. Its study in the low-gravity environment of asteroids is very recent and motivated by the realization that asteroids are covered with regolith. Furthermore, asteroids considered as aggregates can be treated entirely as a granular medium. This domain of research is also motivated by the development of asteroid space missions, such as Hayabusa2 (Watanabe et al. 2017) and OSIRIS-REx (Lauretta et al. 2017), which need simulations of the hardware that will interact with the surface, either to collect samples or to perform in situ measurements. In this regard, the aim of this paper is to contribute to the general understanding of the behavior of granular materials in the low-gravity environment of an asteroid's surface when experiencing an external action, in our case the low-speed interaction of the lander MASCOT on board Hayabusa2.

The JAXA asteroid-sample-return mission Hayabusa2 was launched on December 3, 2014, toward the carbonaceous nearEarth asteroid (162173) Ryugu (Binzel et al. 2001). After arriving at the asteroid in June 2018, the spacecraft intends to carry out a two-month characterization of this asteroid followed by several close approaches to collect some samples and return them to Earth in 2020. Before collecting the desired samples, the main spacecraft will release the European (DLR/CNES) lander MASCOT (Mobile Asteroid Surface Scout) that will perform in situ measurements (Ho et al. 2017) with four instruments including an IR imaging spectrometer (MicrOmega), a 
camera (MASCAM), a radiometer (MARA), and a magnetometer (MASMAG).

The asteroid Ryugu is $870 \mathrm{~m}$ in diameter and although its bulk density is not precisely known yet, its surface gravity is expected to be very low (about $2.5 \times 10^{-4} \mathrm{~m} \mathrm{~s}^{-2}$, Maurel et al. 2017), with an escape speed estimated at about $37 \mathrm{~cm} \mathrm{~s}^{-1}$ (Ho et al. 2017). Such extreme conditions challenge our ability to successfully land a package on the asteroid. The example of Philae, on board the ESA spacecraft Rosetta (Boehnhardt et al. 2017), demonstrated that landing on a low-gravity surface for which we have essentially no a priori information is a great challenge. However, Philae relied on a damping mechanism (which worked) and anchoring (which did not), while MASCOT is designed specifically to bounce. This feature implies that MASCOT's final resting place can be far from the first touchdown point, but operational constraints require keeping that distance small and predicting the area where MASCOT is likely to settle. It is thus important to quantify the outcome of the first impact of the lander as a function of the many parameters of the impact and the environment. Moreover, the traces left by the lander at the impact point can inform us on the surface properties and may also be used to estimate the position and characteristics of the following impact.

This paper addresses these issues with numerical simulations of the interaction between a granular medium (representing the surface of the asteroid) and the lander under expected gravity conditions. The surface of Ryugu is assumed to be covered with a layer of granular material, called regolith, that has been observed on all asteroids for which we have images, and seems to be present on all those that have a thermal inertia estimate (e.g., Delbo et al. 2015). Even if regolith has been observed on asteroids, its actual mechanical properties are poorly known, and we cannot rely on the observations of other asteroids to determine the properties of the Ryugu regolith. For example, even two bodies of the same spectral type, such as the two S-types 433 Eros and 25143 Itokawa, can have very different regolith properties, at least partly due to their very different sizes and therefore gravitational environments. The situation is even worse for C-type asteroids such as Ryugu as to date we have no detailed image of the surface of an asteroid of this type. The only information we have regarding surface properties is an estimate of Ryugu's thermal inertia. According to Müller et al. (2011), the most likely thermal inertia ranges between 200 and $600 \mathrm{~J} \mathrm{~m}^{-2} \mathrm{~K}^{-1} \mathrm{~s}^{-\frac{1}{2}}$, about a factor of 2 lower than the value measured for Itokawa. Within this range, the surface state is expected to go from a thickdust regolith to a boulder/cm-sized, gravel-dominated surface similar to that of 25143 Itokawa. In the absence of the critical parameters necessary to anticipate Ryugu's surface, numerical simulations become a precious resource. Simulations can provide an estimated behavior of the lander for multiple configurations covering a parameter space too large to be experimentally explored in order to be best prepared for the landing phase of the mission.

This study builds on a previous work by Maurel et al. (2017) who performed simulations of MASCOT's landing. Here, we extend the parameter space of those simulations and perform additional data analysis that can help in the interpretation of MASCOT's interaction with the surface and in the search of the lander on the surface if it bounces. Our results could also help the mission's team to infer non-resolved regolith properties from observed ones. The actual landing is scheduled for early October 2018, and this study aims to provide some useful information at the time of landing (on the probable position of MASCOT and on the regolith properties) and also to provide a numerical tool that is already tested and ready to be efficiently used once we are at the asteroid. Moreover, once there, if the actual data on the regolith is out of the range of our present assumptions, new simulations can be run to determine how MASCOT will react on the actual surface.

In Sect. 2, we present the method used to perform the numerical simulations and the considered parameter space for the regolith properties and the impact conditions of the lander. Section 3 presents the results. Conclusions and perspectives are given in Sect. 4.

\section{Methodology}

\subsection{Numerical code: pkdgrav}

The interaction between MASCOT and a granular medium (representing the asteroid's regolith) was simulated with the parallel $\mathrm{N}$-body gravity tree code pkdgrav (Richardson et al. 2000, 2009, 2011; Stadel 2001). To capture the dynamics and contacts between grains of a granular material, the soft-sphere discrete element method (SSDEM) was implemented in the code by Schwartz et al. (2012). We also used the implementation of a new rotational resistance model for the grains (Zhang et al. 2017), which considers twisting and rolling spring-dashpot-slider models.

The pkdgrav code also computes, under any gravitational environment, the interactions between spherical grains and "walls", whose geometry and physical properties (like the distribution of mass within the walls, i.e., the inertia tensor) can be defined. Walls can be assigned as "reactive", meaning they react to forces from the particles (otherwise they are treated as having infinite mass), and can form an "assembly" of multiple walls to represent a sampling device or a lander.

Various experiments have already been used to validate the SSDEM implementation into pkdgrav. For instance, simulation results have been compared with experiments with hopper discharges by Schwartz et al. (2012), low-speed impacts and projectile penetration depths by Schwartz et al. (2014) and Ballouz (2017), and avalanches and angles of repose by Yu et al. (2014) and Maurel et al. (2017). Some preliminary comparisons have also been performed with drop-tower experiments of lander impacts on a regolith bed in low gravity, and show good agreement.

\subsection{Setup of the simulation}

We considered the landing of MASCOT on a regolith bed with an impact speed of $19 \mathrm{~cm} \mathrm{~s}^{-1}$ (upper limit of what is expected for the impact, Biele et al. 2017). The setup of the regolith bed in our simulations is similar to that used by Maurel et al. (2017): a cylindrical non-reactive wall with its top face open, filled with Gaussian size-distributed, spherical soft particles representing the regolith. The gravity considered in our simulations is the same as that assumed for Ryugu, i.e., $2.5 \times 10^{-4} \mathrm{~m} \mathrm{~s}^{-2}$ (Maurel et al. 2017). It is based on the reasonably well-known effective diameter (Müller et al. 2011, 2017) and inputs from JAXA concerning shape, rotation rate, and an estimate of the uncertainty of the center of gravity, and is computed considering simple rotating sphere approximation.

The granular beds are created by letting the particles freefall into the cylinder, letting them relax under the desired level of gravity, and removing any that end up higher than the top of the cylinder. This method ensures a randomness in the position of the grains in the cylinder at the expense of large CPU 
Table 1. Characteristics and properties of the two material types considered in our simulations.

\begin{tabular}{cccccccc}
\hline \hline Material type & Angle of repose $\left(^{\circ}\right)$ & $\varepsilon_{n}$ & $\varepsilon_{t}$ & $\mu_{s}$ & $\mu_{r}$ & $\mu_{t}$ & $\beta$ \\
\hline $\begin{array}{c}\text { Gravel-like friction } \\
\text { Moderate friction }\end{array}$ & $\begin{array}{c}38.5 \\
28\end{array}$ & 0.5 & 0.5 & 1.0 & \multirow{2}{*}{1.05} & \multirow{2}{*}{1.3} & $\begin{array}{c}1.0 \\
0.2\end{array}$ \\
\hline
\end{tabular}

times. Since we do not know in advance the regolith properties, two different types of regolith grains were considered, one with a gravel-like friction and one with a moderate friction. These two frictions differ in their equivalent angle of repose (Table 1), corresponding in the simulations to two different shape factors $\beta$ (Zhang et al. 2017). The shape factor $\beta$ is directly linked to the angle of repose because it represents the angularity of the particles: the higher it is, the more angular the particles are, and therefore particles will slide less effectively on each other, resulting in a steeper angle of repose. The interaction between particles in contact in pkdgrav is mainly controlled by six parameters: the normal and tangential coefficients of restitution $\varepsilon_{n}$ and $\varepsilon_{t}$; the interparticle friction coefficients for sliding, rolling, and twisting, respectively $\mu_{s}, \mu_{r}$, and $\mu_{t}$; and the shape factor $\beta$. Here $\varepsilon_{n}$ and $\varepsilon_{t}$ dominate the energy dissipation, and the other four parameters describe the frictional strength (see Sect. 2 in Zhang et al. 2017 for details). In Sects. 3.6 and 3.7 we analyze the influence of the coefficients of restitution of MASCOT and the grains, respectively.

Regarding the depth of the regolith (the depth of the cylinder), we consider 15,30 , and $40 \mathrm{~cm}$ (Table 2). The depth controls possible boundary effects. If it is shallow enough, the wave produced by MASCOT's impact will reflect at the bottom, representing a situation where a hard surface is covered by a thin layer of regolith. The deeper case $(40 \mathrm{~cm})$ represents a surface with a layer of regolith that is thick enough that the wave produced by the impact almost never sees a hard bottom, and when it does, by the time it comes back to the surface, the lander has already bounced away. This has been confirmed by changing the properties of the bottom wall for the different depths considered. For further information about the influence of the bed depth, see Sect. 3.3. The diameter of the cylinder is $150 \mathrm{~cm}$, corresponding to a little more than five times the largest dimension of MASCOT (i.e., $29 \mathrm{~cm}$ ). When the diameter of the container is less than five times the diameter of the projectile, according to Seguin et al. (2008), some boundary effects may play a role. However, according to Goldman \& Umbanhowar (2008), for impact speeds low enough (less than $2 \mathrm{~m} \mathrm{~s}^{-1}$ ), the diameter of the cylinder should not have a significant effect on the results. Nonetheless, we used to methods to check that the outermost particles do not feel the impact, or at least do not influence the fate of the lander or the crater attributes. First, throughout the simulations we checked the maximum and root mean square (RMS) speeds of particles contained in the outermost rectangular cross section torus whose width is $5 \mathrm{~cm}$ and height is that of the cylinder. The RMS speed never goes higher than $0.06 \mathrm{~cm} \mathrm{~s}^{-1}$, which is too low to influence the lander's behavior. A lone particle can have a speed of up to $2.5 \mathrm{~cm} \mathrm{~s}^{-1}$, but does not create feedback on the lander or the crater after the wave bounces on the cylindrical wall. Second, we modified the coefficients of restitution of the cylindrical wall and did not observe any meaningful variations in the results.

In terms of size distribution of the grains, since there are no well-known constraints, we used a Gaussian distribution of particle radii with mean radius of $1 \mathrm{~cm}$, a standard deviation
Table 2. Different depths considered in our simulations with the corresponding numbers of particles for the two different material types.

\begin{tabular}{ccc}
\hline \hline Depth & Gravel-like friction & Moderate friction \\
\hline $15 \mathrm{~cm}$ & 28375 particles & 31082 particles \\
$30 \mathrm{~cm}$ & 58454 particles & 62551 particles \\
$40 \mathrm{~cm}$ & 78176 particles & 83097 particles \\
\hline
\end{tabular}

(sigma) of $33 \%$, and a cut-off after $1 \sigma$. This assumption represents one of the possible cases derived from the range of thermal inertia estimated for the asteroid (Gundlach \& Blum 2013), although we recognize that it is idealized compared with a more realistic power-law size distribution. The influence of a powerlaw size distribution was succinctly treated by Maurel et al. (2017), who found that it mostly enhances the stochastic aspect of the simulations. However, we did not consider it here because it would significantly increase the computation time of every simulation.

The numerical model of MASCOT is described in Maurel et al. (2017). It consists of a $19.5 \mathrm{~cm} \times 27.5 \mathrm{~cm} \times 29 \mathrm{~cm}$ cuboid, with a small prominence representing the sensor of the hyperspectral microscopic imager (MicrOmega). MASCOT and MicrOmega form what we called earlier an assembly of reactive walls, and is initially placed about $40 \mathrm{~cm}$ above the top of the regolith bed.

The initial (slow) rotation of MASCOT after ejection from the Hayabusa2 spacecraft, which is the result of various nonideal conditions, has a large dispersion. With the uncertainties on gravity (and thus on the duration of the descent) and on the release height above the actual first contact point, all these dispersions make the attitude at first contact random. However, MASCOT is more likely to land on a corner, and because the impact orientation is just one of the many parameters that can have an effect and that we need to study, we decided to focus on three possible orientations (see Fig. 1): Flat, Back-CornerFirst (BCF) and Front-Corner-First (FCF). Indeed, MASCOT is more likely to land on a corner and, since we wanted to study the effect of many different parameters and not only orientation, we considered only these three orientations. Other orientations, for example MASCOT landing on an edge, have been studied by Maurel et al. (2017). A slow spin like that transmitted to the lander during its ejection from the spacecraft $\left(0.1 \mathrm{rad} \mathrm{s}^{-1}\right)$ does not induce any critical change in the outcomes of the impact (Maurel et al. 2017). We therefore considered no initial spin in our simulations.

Since, as we said previously, there are still a lot of uncertainties concerning MASCOT's separation, the landing site, and Ryugu's surface topography, the range of MASCOT's angle of impact is broad. However, to study the influence of the impact angle, we restrained it to five different angles, as done by Maurel et al. (2017): $0^{\circ}, 15^{\circ}, 30^{\circ}, 45^{\circ}$, and $60^{\circ}\left(0^{\circ}\right.$ means a purely vertical trajectory; larger angles represent more grazing impacts). 


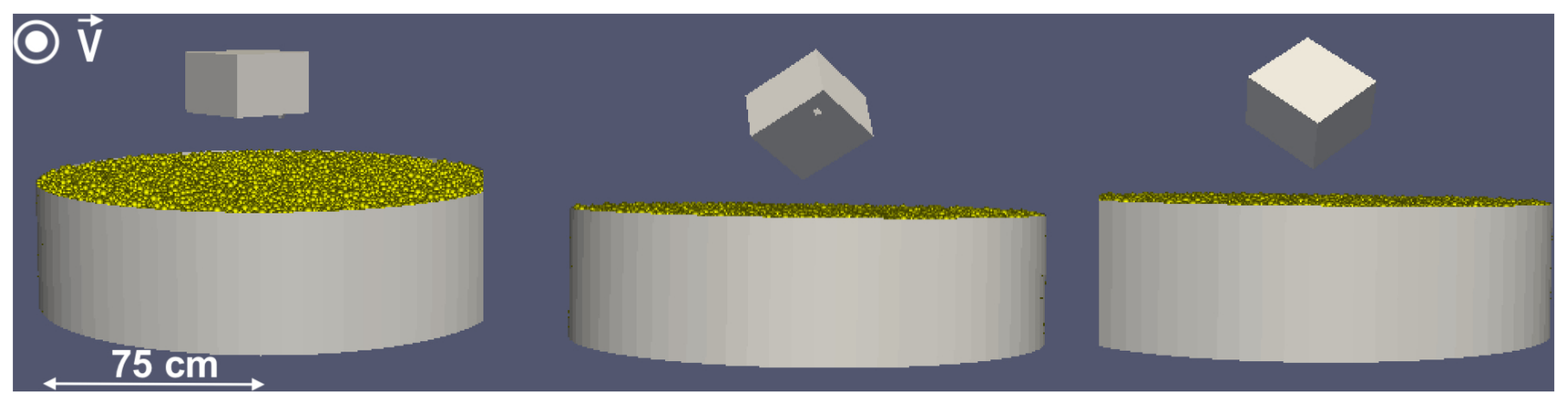

Fig. 1. Different orientations of MASCOT (coming toward the reader in these snapshots) used in our simulations: Flat, Back-Corner-First, and Front-Corner-First

\subsection{Investigated output quantities}

We can compute several characteristics related to the impact itself: the outgoing-to-incoming speed ratio of MASCOT's center of gravity, the outgoing rotational-to-linear energy ratio, the collision duration, the maximum penetration depth of the lander into the bed, and the outgoing trajectory angle. The maximum penetration depth corresponds to the lowest point reached by any of the corners of MASCOT, and the collision duration the time from MASCOT's first contact with the regolith bed until all its height corners are above the plane at the top of the cylinder. These parameters were chosen because they enable us to describe the collision (duration, penetration of the lander into the regolith bed) as well as MASCOT's state just after the collision (outgoing speed, energies, and trajectory angle).

We also compute the evolution of MASCOT after its first impact. If MASCOT bounces after its first impact, we ballistically extrapolate its future behavior as a free-fall trajectory, using the data from the end of the simulation of the first impact. Doing so, we can determine the distance that MASCOT travels after the first impact, the time between the first and the second impact, and the second impact speed and angle, considering a flat environment. The distance traveled by MASCOT is the distance on the surface (assumed to be flat) between the spot where MASCOT leaves the ground (its height corners are higher than the surface) and the spot where MASCOT touches the ground again (when its equivalent spherical radius reaches the surface). The distance traveled by MASCOT is mostly directed by the outgoing speed and the outgoing angle between MASCOT's motion direction and the horizontal plane, but not necessarily. The ejected particles impacting the bottom of MASCOT when leaving the bed may indeed affect its trajectory. The simulations are long enough to allow a good extrapolation of MASCOT's behavior after the impact.

Furthermore, we studied the properties of the crater resulting from the first impact, particularly its shape and depth. At the end of our simulations, the crater may still be transient, which is the state in which it will probably be observed by the Hayabusa2 team just after the landing of MASCOT. The aim of this analysis is to understand whether the transient crater's properties can be used as a diagnostic of MASCOT's distance from the impact point, provided that the camera on board Hayabusa2 can resolve it and that we find a direct link between those properties and MASCOT's fate in our simulations. As we will see, those properties can also give us some information on the regolith properties, as different properties yield different traces.

The Hayabusa2 optical navigation camera (ONC) system, which consists of a telescope (ONC-T) and two wide-angle cameras (ONC-W1 and -W2) (Kameda et al. 2017; Suzuki et al. 2018) can observe MASCOT while it is descending as well as crater(s) and ejecta deposits resulting from MASCOT's impact. During Hayabusa2's ascending sequence following the release of MASCOT, the slant viewing ONC-W2 camera will obtain images of separation motion of MASCOT from the side panel of the Hayabusa2 main spacecraft. Then, the nadir-viewing ONC-W1 camera will take images of MASCOT's fall. These observations will help estimate MASCOT's trajectory before the first bounce. As shown in Sect. 3.1, the angle and velocity of MASCOT's impact will be determined by MASCOT's descent trajectory and Ryugu's local topography and will provide constraints on Ryugu's surface physical properties. Subsequently, ONC-T will start taking a sequence of images of the surface area around MASCOT's first bounce location. The spatial resolution and field of view (FOV) of the ONC-T camera will change due to the ascent of Hayabusa2, which may depend on many unknown parameters of Ryugu, such as the surface gravity. We note that the nominal spatial resolution and FOV of each ONC-T image are about $3.6-11 \mathrm{~cm} \mathrm{pix}^{-1}$ and $36-108 \mathrm{~m}$, respectively. This resolution should be good enough to capture and characterize the traces left by MASCOT after its impact on the asteroid because the typical crater diameter in our simulations is between 160 and $200 \mathrm{~cm}$. Also, because the total coverage of the ONC-T images taken during the ascent is $150-200 \mathrm{~m}$ in diameter, it should cover the landing ellipsoid of MASCOT's first bounce, which is expected to be of the same order of magnitude as MASCOT's releasing altitude $(\sim 60 \mathrm{~m})$. However, the total number of such high-resolution ONC-T images is limited to nine in the current plan; this series of images may end before comes to rest after multiple bounces. Subsequent imaging of MASCOT will be performed from much higher altitudes $(\sim 3 \mathrm{~km})$ with lower resolutions $\left(\sim 30 \mathrm{~cm} \mathrm{pix}^{-1}\right)$. If it bounces, finding MASCOT on the asteroid may thus become a challenge, as it was for Philae. It may be easier to find MASCOT's first impact site in the ellipse of uncertainty, when the main spacecraft is still at low altitudes, and to image the traces. We therefore also investigated the relations between the distance traveled by the lander and the traces left in the ground.

\section{Outcomes of the lander/regolith interaction and sensitive parameters}

In this section, we derive general trends from our simulations in terms of traveled distance of the lander after the first impact, traces left on the asteroid surface, and other outcomes, and identify the sensitive parameters that drive these trends. 


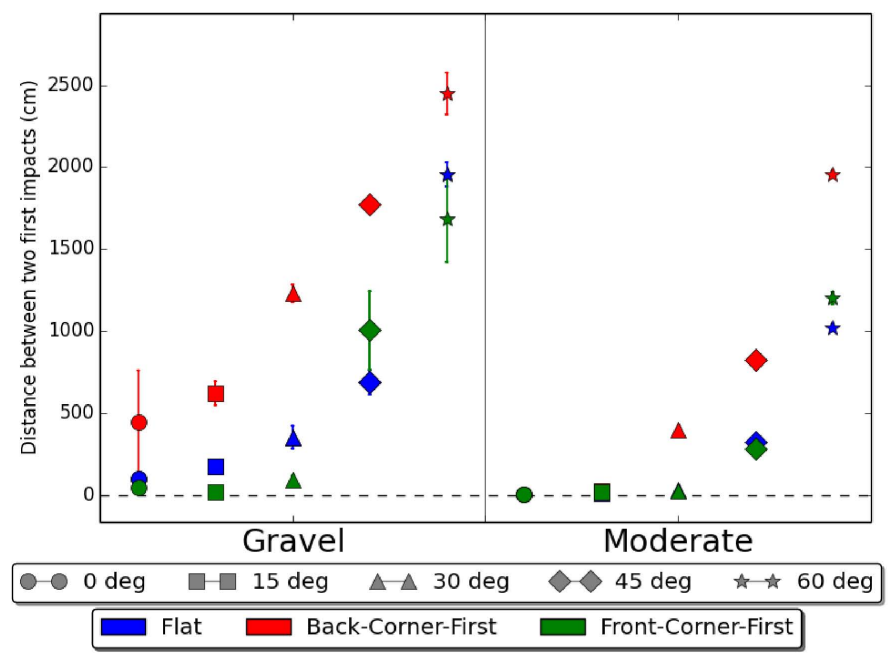

(a) $30 \mathrm{~cm}$ deep bed

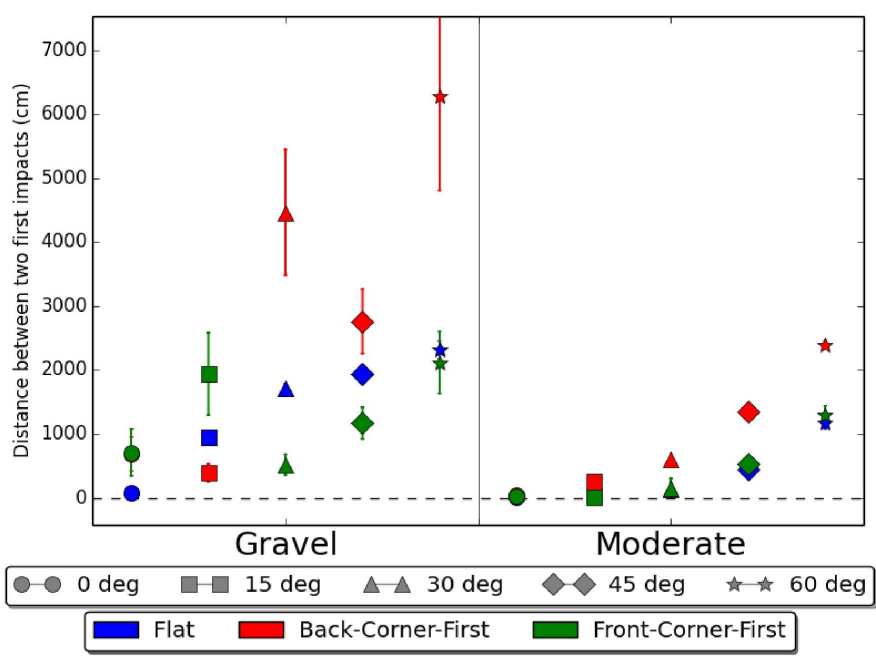

(b) $15 \mathrm{~cm}$ deep bed

Fig. 2. Distance traveled by the lander between the first bounce and the second impact as a function of the impact angle (" 0 deg" means a pure vertical trajectory with no lateral motion; larger angles represent more grazing impacts), the material type, and the orientation of MASCOT at impact, for a $30 \mathrm{~cm}$ bed (left) and a $15 \mathrm{~cm}$ bed (right). The shapes of the markers represent the five angles considered, while the two columns show the two material types. The color refers to the orientation of MASCOT. The arrows represent error bars (see text for details). When the error bars are not visible, they are smaller than the markers.

Table 3. Average impact characteristics for 54 simulations with three different depths, two material types, and three orientations at impact for each of the five different angles of approach considered in our simulations.

\begin{tabular}{|c|c|c|c|c|c|c|c|c|c|}
\hline Angle & $\begin{array}{l}\text { Traveled } \\
\text { distance }\end{array}$ & $\begin{array}{c}\text { Time between } \\
\text { impacts }\end{array}$ & $\frac{V_{\text {out }}}{V_{\text {in }}}$ & $\frac{E_{\mathrm{rot}, \mathrm{out}}}{E_{\text {lin,in }}}$ & $\frac{E_{\text {rot,out }}}{E_{\text {lin,out }}}$ & $\begin{array}{c}\text { Collision } \\
\text { duration }\end{array}$ & $\begin{array}{c}\text { Maximum } \\
\text { penetration depth }\end{array}$ & $\begin{array}{l}\text { Incoming angle } \\
\text { at second impact }\end{array}$ & $\begin{array}{c}\text { Speed at } \\
\text { second impact }\end{array}$ \\
\hline $0^{\circ}$ & $1.2 \mathrm{~m}$ & $84 \mathrm{~s}$ & $6 \%$ & $1.9 \%$ & $359 \%$ & $11.0 \mathrm{~s}$ & $9.4 \mathrm{~cm}$ & $32^{\circ}$ & $1.2 \mathrm{~cm} \mathrm{~s}^{-1}$ \\
\hline $15^{\circ}$ & $2.7 \mathrm{~m}$ & $86 \mathrm{~s}$ & $11 \%$ & $1.1 \%$ & $142 \%$ & $10.6 \mathrm{~s}$ & $8.5 \mathrm{~cm}$ & $48^{\circ}$ & $1.6 \mathrm{~cm} \mathrm{~s}^{-1}$ \\
\hline $30^{\circ}$ & $6.0 \mathrm{~m}$ & $153 \mathrm{~s}$ & $17 \%$ & $3.0 \%$ & $54 \%$ & $9.5 \mathrm{~s}$ & $7.9 \mathrm{~cm}$ & $54^{\circ}$ & $2.9 \mathrm{~cm} \mathrm{~s}^{-1}$ \\
\hline $45^{\circ}$ & $10.1 \mathrm{~m}$ & $231 \mathrm{~s}$ & $26 \%$ & $5.0 \%$ & $58 \%$ & $6.1 \mathrm{~s}$ & $5.9 \mathrm{~cm}$ & $56^{\circ}$ & $4.9 \mathrm{~cm} \mathrm{~s}^{-1}$ \\
\hline $60^{\circ}$ & $19.7 \mathrm{~m}$ & $295 \mathrm{~s}$ & $41 \%$ & $7.4 \%$ & $45 \%$ & $3.8 \mathrm{~s}$ & $5.2 \mathrm{~cm}$ & $61^{\circ}$ & $7.4 \mathrm{~cm} \mathrm{~s}^{-1}$ \\
\hline
\end{tabular}

Notes. $V, E_{r o t}$, and $E_{\text {lin }}$ correspond respectively to MASCOT's speed, rotational energy and linear energy, and out and in to outgoing and incoming values.

\subsection{Influence of the angle of impact}

One of the most influential parameters on the distance traveled by the lander is the angle of impact. In our simulations, we notice an increase in the distance traveled by MASCOT after the first impact correlated with the angle of impact. In other words, the more grazing the impact, the greater the traveled distance. The process governing the impact is described in detail in Sect. 3.2, but the higher the tangential component of the velocity, the higher the tangential speed after the impact, and the higher the bouncing probability, which leads to a greater distance traveled. These results are clearer for regolith beds of $30 \mathrm{~cm}$ and $40 \mathrm{~cm}$ in depth, as shown in Fig. 2a. For a $15 \mathrm{~cm}$ bed (Fig. 2b), due to the boundary effects of the bottom of the bed (see Sect. 3.3 for the influence of depth), the points are more scattered and the trend is less obvious, but it holds in a general way. The error bars correspond to standard deviations obtained for three similar simulations. The stochasticity in the simulations is more thoroughly described in Sect. 3.8, but we note that it does not invalidate the trends established here. Therefore, and for better readability, we do not include error bars in every figure.

Table 3 displays an average of several characteristic output quantities over the different considered bed depths, material types, and orientations of MASCOT (54 simulations for each of the five angles of impact considered here, for a total of 270 simulations). Once again, we note that the more grazing the impact (i.e., the higher impact angle), the greater the distance traveled. When the impact angle is high, the lander spends less time in contact with the soil, the collision is shorter, and less energy is imparted to the bed particles (resulting in a shallower crater, if we consider the maximum penetration depth). However, recalling that the incoming linear energy is the same for all simulations, we note that the outgoing rotational energy increases with the angle of impact, whereas the outgoing rotational-tolinear energy ratio sharply decreases when the impact angle increases. This indicates that for grazing angles, the impact makes MASCOT spin more than for vertical ones, but the increase in rotational energy is less significant than the increase in linear energy when the angle of impact increases. Moreover, the outgoing rotational energy is always much lower than the incoming linear energy, but the outgoing linear energy depends largely on the angle of approach. After the impact, depending on the angle, the linear energy can be either dominant or dominated over the rotational energy: for vertical impact, the rotational energy is higher than the linear energy, whereas it is the opposite for very grazing impacts. 


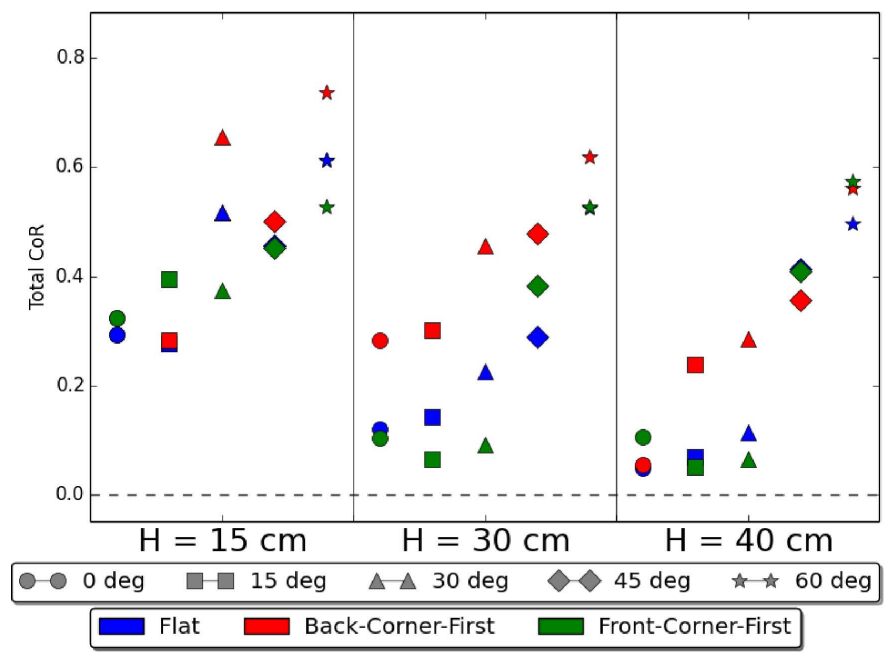

(a) With gravel-like friction

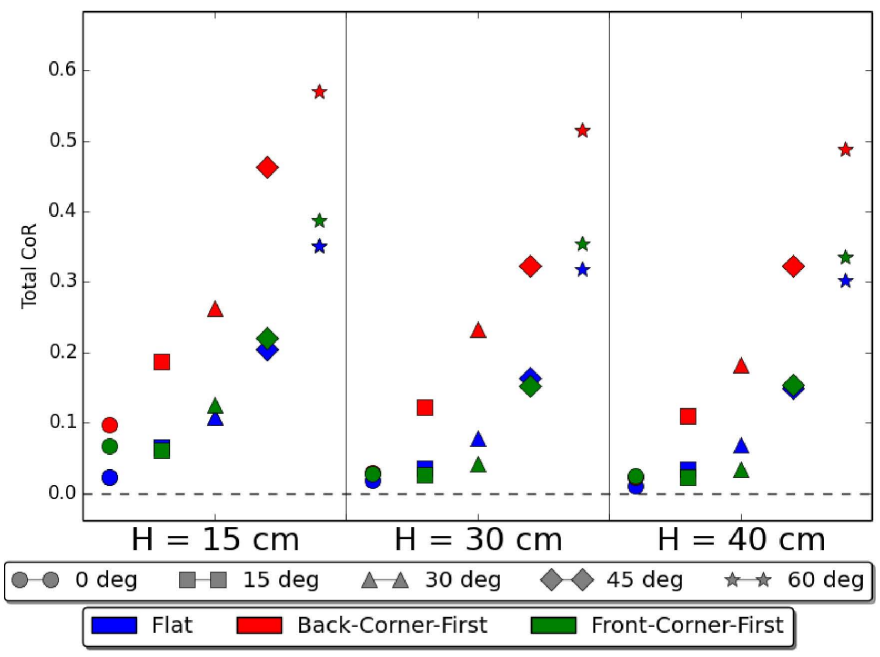

(b) With moderate friction

Fig. 3. Total coefficient of restitution CoR as a function of the impact angle (" 0 deg" means a pure vertical trajectory with no lateral motion; larger angles represent more grazing impacts), the bed depth, and the orientation of MASCOT at impact, for a gravel-like friction (left) and a moderate friction $(r i g h t)$. The shapes of the markers represent the five considered angles, while the three columns show the three bed depths. The color refers to the orientation of MASCOT.

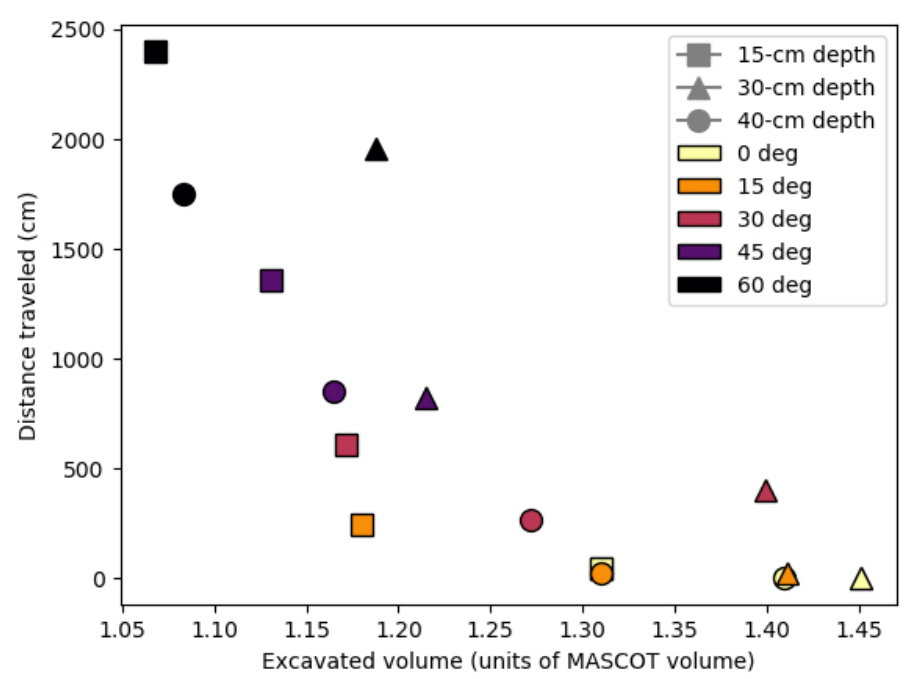

Fig. 4. Excavated volume vs. distance traveled by the lander between the first bounce and the second impact at the end of the simulation as functions of the impact angle and the bed depth. Only the Back-CornerFirst MASCOT impact orientation is shown, with a moderate-friction regolith. The shade of gray depends on the impact angle and the symbol shape on the bed depth, as shown in the legend. MASCOT volume is about $1.56 \times 10^{4} \mathrm{~cm}^{3}$.

Figure 3 shows MASCOT's outgoing-to-incoming total energy ratio (also called total coefficient of restitution, or total CoR) considering both rotational and linear energies for different bed depths, angles of impact, orientations, and frictions. We note that the total CoR increases when the angle of impact increases (i.e., when the impact is more grazing), as does the traveled distance.

We also analyzed the influence of the angle of impact on the ejected volume of regolith. As shown in Fig. 4, a grazing impact ejects less material, and makes a shallower crater. However, these trends are only present for angles higher than $30^{\circ}$ and for a moderate-friction regolith. For gravel-like material, there is no clear trend as the impact angle increases (see Fig. 5) and we show in Sect. 3.2 the differences in the mechanisms of the

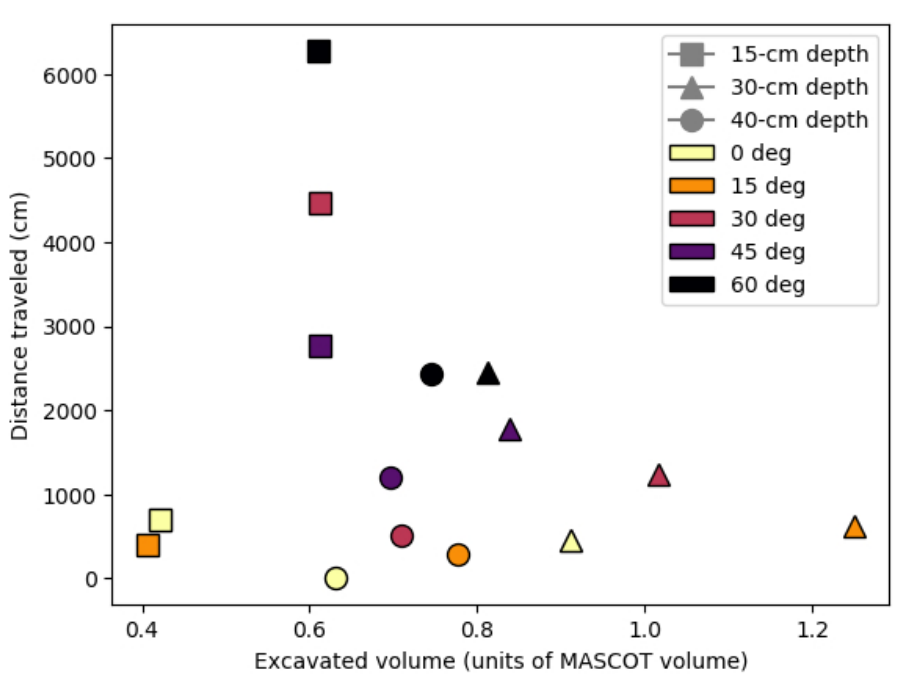

Fig. 5. Excavated volume vs. distance traveled by the lander between the first bounce and the second impact at the end of the simulation as functions of the impact angle and the bed depth. Only the Back-CornerFirst MASCOT impact orientation is shown, with a gravel-like regolith. The shade of gray depends on the impact angle and the symbol shape on the bed depth, as shown in the legend. MASCOT volume is about $1.56 \times 10^{4} \mathrm{~cm}^{3}$.

impact between the different types of regolith considered in this paper. These differences of excavated volume and penetration depth are visible in Fig. 6, where we compare the craters formed after impacts at $15^{\circ}$ and $45^{\circ}$. The depth of the crater may give hints to determine the first angle of impact and thus the distance traveled by MASCOT after the first bounce.

In the event of a second impact, the incoming speed as well as the angle of the second impact strongly increase with the angle of the first impact (Table 3). It is mainly due to MASCOT having higher tangential velocities before grazing impacts than before normal ones and therefore having a slightly higher tangential velocity after the impact, leading to higher second impact angles. Peaks of occurrence for the five different considered angles are shown in Fig. 7. Even if we can see a slight increase with the 

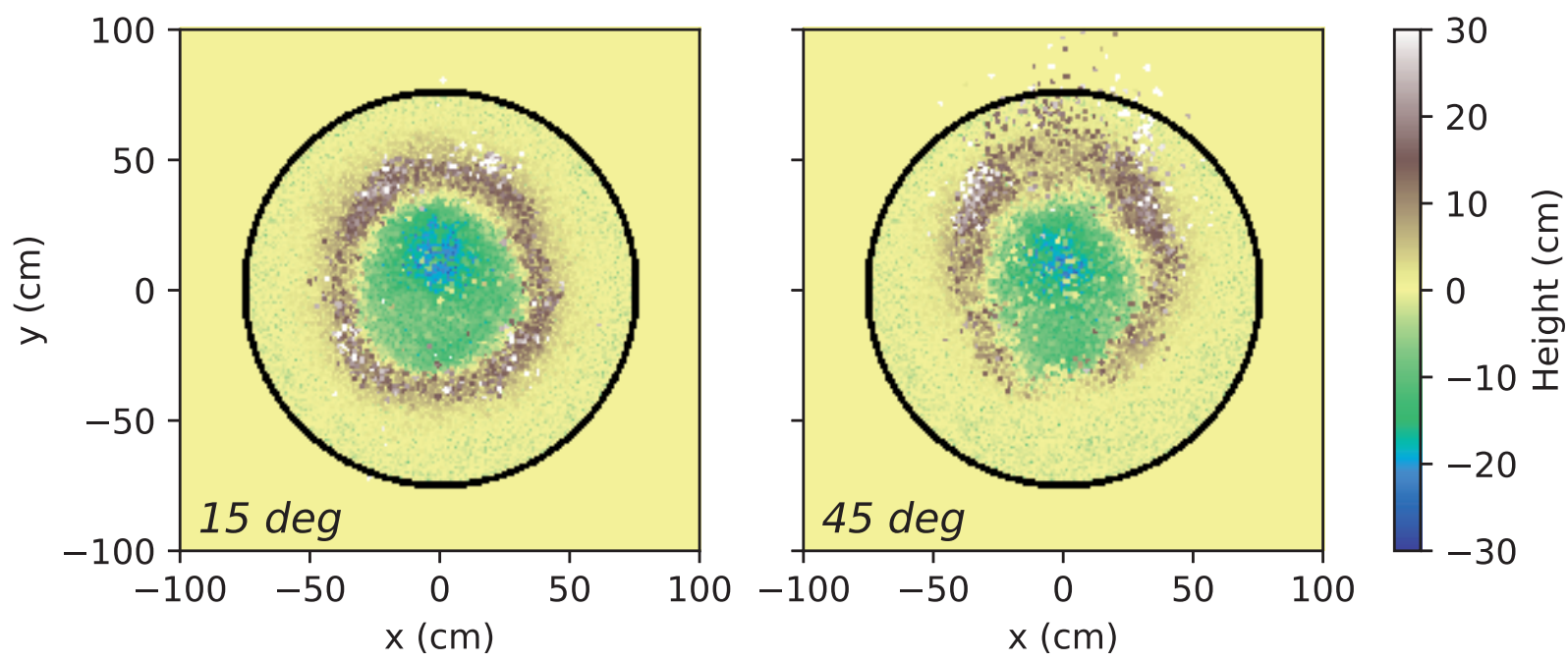

Fig. 6. Relief of the trace left by MASCOT on the regolith about $14 \mathrm{~s}$ after the impact for a moderate-friction regolith. The color bar represents the height of the regolith (bed and ejecta). MASCOT lands in the Back-Corner-First configuration with an impact angle of $15^{\circ}$ (left) and at $45^{\circ}($ right). The regolith is $30 \mathrm{~cm}$ deep. For the $15^{\circ}$ impact, MASCOT has not left the surface (its height corners are still under the surface level), whereas it did after $7.5 \mathrm{~s}$ for the $45^{\circ}$ impact.

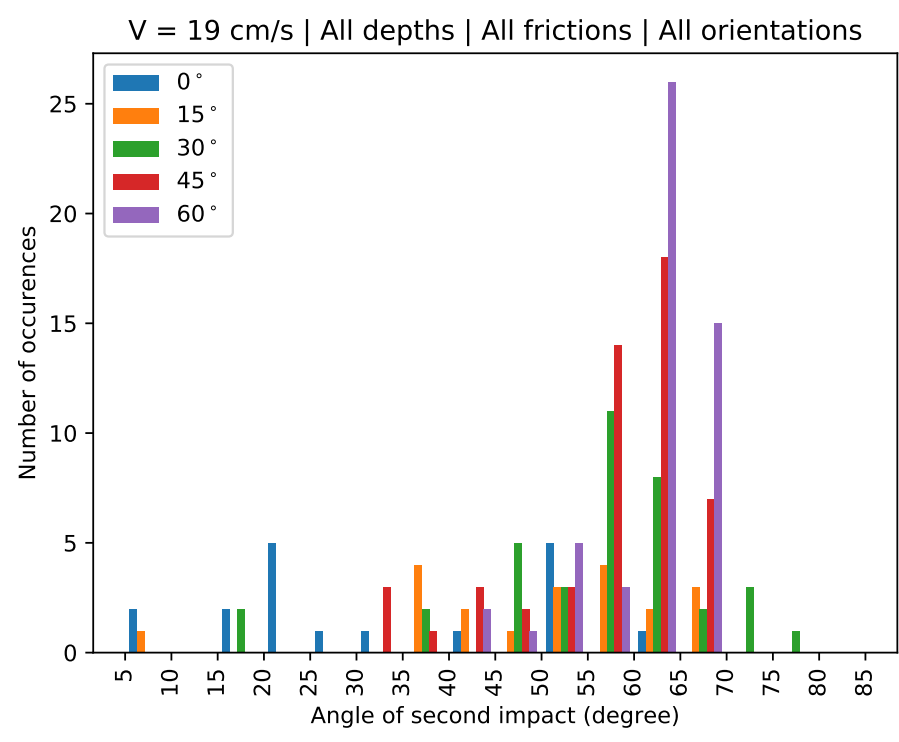

Fig. 7. Histogram of the second impact angle in the event of a second contact, for the five angles of impact considered in this article. The data represent the five angles of impact, the three bed depths, the three orientations, and the two friction properties (total of 270 simulations).

first impact angle, the second impact angle is mostly around $60^{\circ}$. Indeed, when MASCOT bounces at the first impact, almost $45 \%$ of the simulations show a second impact angle between $55^{\circ}$ and $65^{\circ}$, and more than $65 \%$ show one between $50^{\circ}$ and $70^{\circ}$. Regolith friction, cylinder depth and MASCOT's orientation show no significant influence on the second impact angle directly.

The high value of the second impact angle may lead to a second bounce; we could assume that it would be weaker because of the smaller impact speed, but we also have to consider the role of the rotation after the first bounce. As we already indicated, spins of $0.1 \mathrm{rad} \mathrm{s}^{-1}$ have already been studied by Maurel et al. (2017). However, we do see in our simulations that the final spin of the lander can be greater than $0.1 \mathrm{rad} \mathrm{s}^{-1}$ (Fig. 8 shows that it can reach more than $0.8 \mathrm{rad} \mathrm{s}^{-1}$ ) and more work remains to be done to understand whether a fast spin can influence the

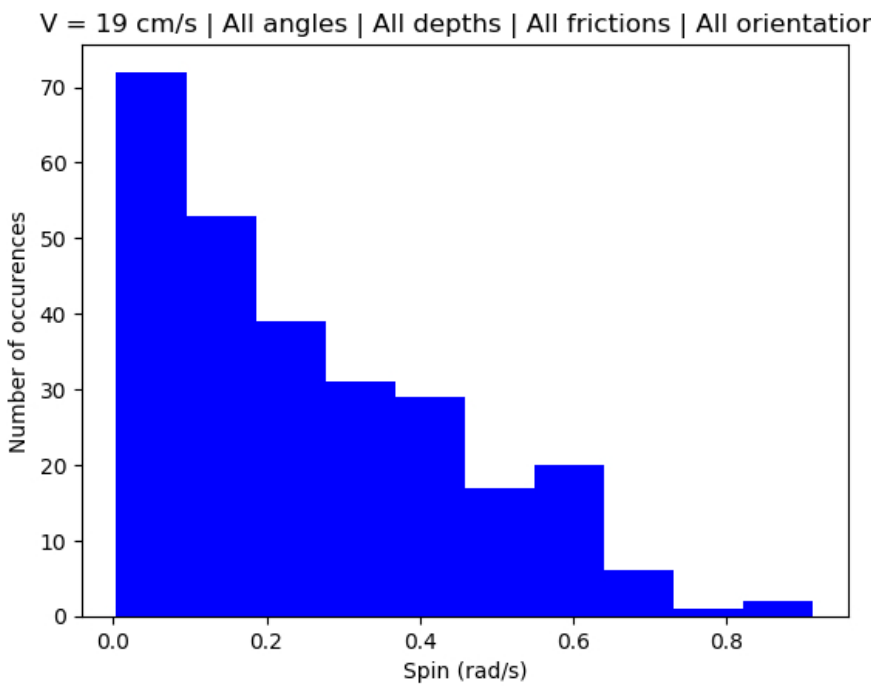

Fig. 8. Histogram of the final spin after the first impact. The data represent the five angles of impact, the three bed depths, the three orientations, and the two friction properties (total of 270 simulations).

putative second impact. The resulting spin after the impact can lead to high total coefficients of restitution, as shown in Fig. 9. The four parameters (first impact angle, regolith friction, depth of the bed, and MASCOT's orientation) influence the spin, the total CoR, and the traveled distance equally. Therefore, considering the first impact angle, the more grazing the angle, the higher the spin and the total CoR, and the greater the traveled distance.

\subsection{Influence of the regolith friction properties}

The nature of the regolith, through its friction properties, also has an influence on the different output quantities. As shown in Fig. 2, the distance traveled by MASCOT after the first impact is greater with a gravel-like regolith bed than with a moderatefriction one, in particular for grazing impacts that lead to the greatest distances. Indeed, with a moderate-friction bed, the global resistance is weaker since particles are smoother, allowing the lander to penetrate deeper into the bed, losing more energy 


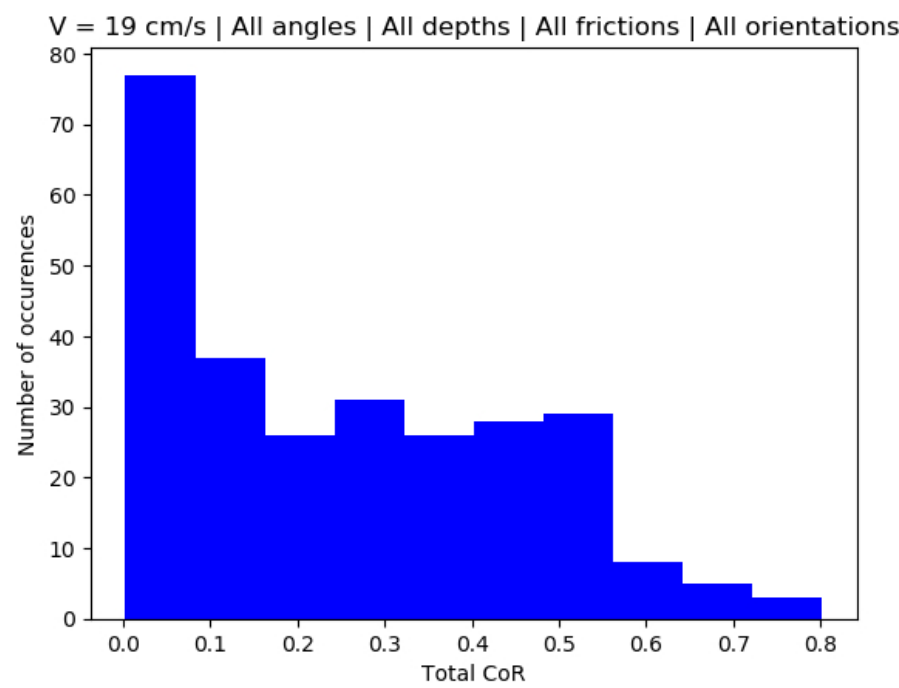

Fig. 9. Histogram of the total CoR (i.e., outgoing-to-incoming total energy ratio). The data represent the five angles of impact, the three bed depths, the three orientations, and the two friction properties (total of 270 simulations).

than it would in a gravel-like medium. Thus, more energy is lost at impact and the lander goes less far.

By looking at cross-sections of the bed during the impact, we can better understand the process governing the impact. Figure 10 shows cross-sections for the Back-Corner-First configuration and we see that, with a gravel-like regolith, the lander hits first with the back corner then pivots swiftly to hit with the front corner. Therefore, the bottom-back part of the lander still has a certain energy and, after its bottom-front part impacts the regolith, its inertia causes the whole body to spin. The bottom face of MASCOT cannot push the regolith particles downward, or at least not enough to penetrate deeply into the bed, because the gravel-like friction makes the bed somewhat rigid. There are in that case two holes in the bed after $30 \mathrm{~s}$. Conversely, the moderate friction makes the penetration smoother, and the lander leaves only one hole. The smaller friction between the particles of the bed makes the bed more compliant, and then MASCOT pivots less but digs more into the regolith. Since the lander pivots more swiftly for a gravel-like regolith bed, the resulting spin after the impact is on average higher than for a moderate-friction bed. The spin and linear speed being higher, the total CoR is also higher.

The impact process described here is particularly true for the Back-Corner-First orientation. The process is similar for the two other configurations (Flat and FCF impacts), that are described in Sect. 3.4. For all the processes discussed in this article, the friction of the regolith bed has the same influence: the higher the friction, the higher the grains' resistance to MASCOT's penetration, and the greater the distance traveled. With gravel-like regolith, the distance is greater, the collision duration is shorter, and the penetration is shallower.

These differences in behavior between the two considered frictions are visible if we look at the characteristics of the traces left by MASCOT. Figures 11 and 12 show snapshots of the regolith bed seen from above, during the landing of MASCOT with an angle of impact of $45^{\circ}$ on the back corner, for a gravellike bed and for a moderate-friction bed, respectively. We notice that the impact of the lander in the gravel-like material bed leaves a two-hole crater, whereas the smoother regolith with moderate friction leads to a one-hole crater, as suggested by the cross-sections. We also notice that the volume of ejecta for the moderate-friction bed is much larger than that produced with a gravel-like bed because the lander gives more energy to the particles, and the particles dissipate less energy among themselves by friction. We find that the volume of ejecta for a moderate-friction bed is on average $80 \%$ larger than that for a gravel-like regolith bed when MASCOT impacts on the back corner, and $60 \%$ when it lands flat on the ground. Therefore, the images taken by the main spacecraft after impact can inform us indirectly, either from the volume of ejecta or from the crater's shape, of the properties of the regolith layer on Ryugu.

Since MASCOT leaves a two-hole crater in the ground for a gravel-like regolith, we can estimate in which direction MASCOT is traveling after the impact by looking at the alignment of the two holes, even if it is not obvious and there is scattering. In Figs. 11 and 12, the lander comes from the bottom and continues toward the top. Also, in the example of the moderate-friction case, the spatial distribution of ejecta informs us about the direction of the lander. Indeed, there's almost no ejecta in the direction MASCOT comes from, and the ejecta travels farther in the direction the lander is going.

After the lander bounces on a moderate-friction bed, since it loses more energy during the bouncing, the incoming speed for the second impact is smaller than that for a gravel-like regolith and therefore we can expect a smaller second bounce or no bounce at all.

In conclusion, the regolith friction has a direct impact on the distance traveled by the lander and on the traces left on the ground (crater size, ejecta volume) and by looking at the size of the crater we can extrapolate MASCOT's direction and distance (for details on the different cases, see Sect. 3.4). However, for scientific purposes, the shape of the traces can also give us precious information about the friction of the regolith, and therefore partially on its nature. Moreover, a posteriori, after we find the lander, its distance from the first impact site may also give us insight on the regolith friction. Obviously, this method cannot give us the exact composition of the regolith directly, but in the event of dysfunction of instruments on board MASCOT or combined with the measurements of these instruments, it can help to deduce the nature of the regolith.

\subsection{Influence of the depth of the regolith bed}

Since the depth of the regolith bed is difficult to measure from distant observations, we also analyzed its influence to determine whether we can infer it from the impact outcome. Table 4 shows averages of several characteristic output quantities over the different considered impact angles, orientations of MASCOT, and material types (each quantity is averaged over 90 simulations for each depth). We see that the shallower the bed, the greater the distance traveled by the lander after the first bounce, and the smaller the penetration depth. We also find that the differences in average impact characteristics are smaller for beds with depths of $30 \mathrm{~cm}$ and $40 \mathrm{~cm}$, implying that the differences seen for the bed with a depth of $15 \mathrm{~cm}$ are largely due to boundary conditions, i.e., to the effect of the bottom of the cylinder. To confirm this, we used different coefficients of restitution for the bottom wall for the different bed depths. We noticed non-negligible variations in the output quantities only for the $15 \mathrm{~cm}$ bed. For a shallow bed, the wave provoked by the impact of the lander on the top of the bed is not damped yet when it reaches the bottom of the cylinder, and reflects off the bottom wall.

Figure 13 shows the root-mean-square (RMS) speed of the particles in each $5 \mathrm{~cm}$ layer forming the regolith bed. It can be 

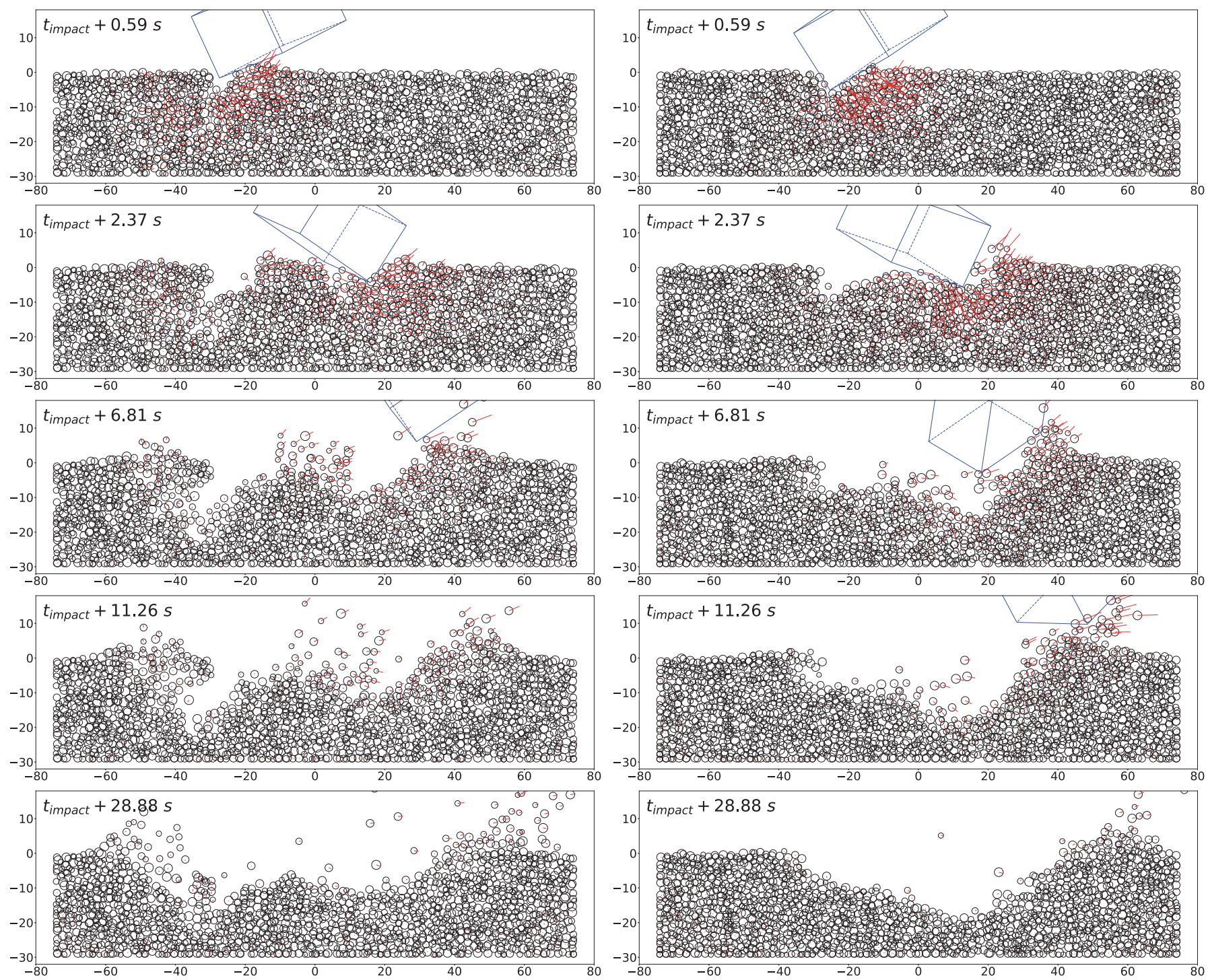

Fig. 10. Snapshots of MASCOT impact, represented in a cross-section of a gravel-like regolith bed (left column) and a moderate-friction regolith bed (right column) for different times. The blue cuboid represents MASCOT, and each red line indicates the projected 2D velocity of the corresponding particle. MASCOT lands in the Back-Corner-First configuration with an impact angle of $45^{\circ}$, and the regolith is $30 \mathrm{~cm}$ deep.

noticed that for a $40 \mathrm{~cm}$ bed, the wave almost does not reach the bottom, and therefore will not reflect off it, whereas for a $15 \mathrm{~cm}$ bed the particles at the bottom of the cylinder are submitted to relatively high velocities.

Considering the second bounce, as the regolith layer gets thicker, the speed at second impact decreases (Table 4) and the total expected number of lander bounces decreases. The afterimpact spin and the total CoR also decrease when the regolith layer is deeper.

\subsection{Influence of the orientation}

As shown in Fig. 2, the distance traveled by MASCOT in the Back-Corner-First configuration is greater than that in the two other configurations. The way MASCOT hits the granular medium influences the dynamics of the lander. In the BackCorner-First configuration, because the first contact point is behind MASCOT's center of gravity (in the direction of motion), the resulting rotation is in the same direction as the initial motion of MASCOT, and therefore the lander is only partly slowed down. On the other hand, for the Front-Corner-First configuration, MASCOT's center of gravity is behind the first contact point, which results in a greater slowdown and therefore a shorter traveled distance. The two configurations show very similar impact outcomes, but the $\mathrm{BCF}$ orientation has a first phase: when the back corner impacts the ground, MASCOT gains spin and momentum and therefore impacts in a second phase with its front corner with a higher momentum. With the FCF orientation, the front corner impacts the ground directly with no spin beforehand and the traveled distance is consequently shorter.

When MASCOT lands flat on the regolith bed, the process is the same as for the two other orientations. Since the ground is not completely flat, the lander cannot land exactly on its bottom side. It also cannot land perfectly on an edge, and either one of the back corners or the front corners will touch the bed first, leading to the previous cases but in a softer way. However, since the first contact with the ground may be close to a corner and not exactly on one of these, and since it is not oriented the same way as for BCF and FCF (i.e., back and front corners aligned with the tangential velocity) the distance traveled after MASCOT landed flat may even be smaller than with FCF orientation. 

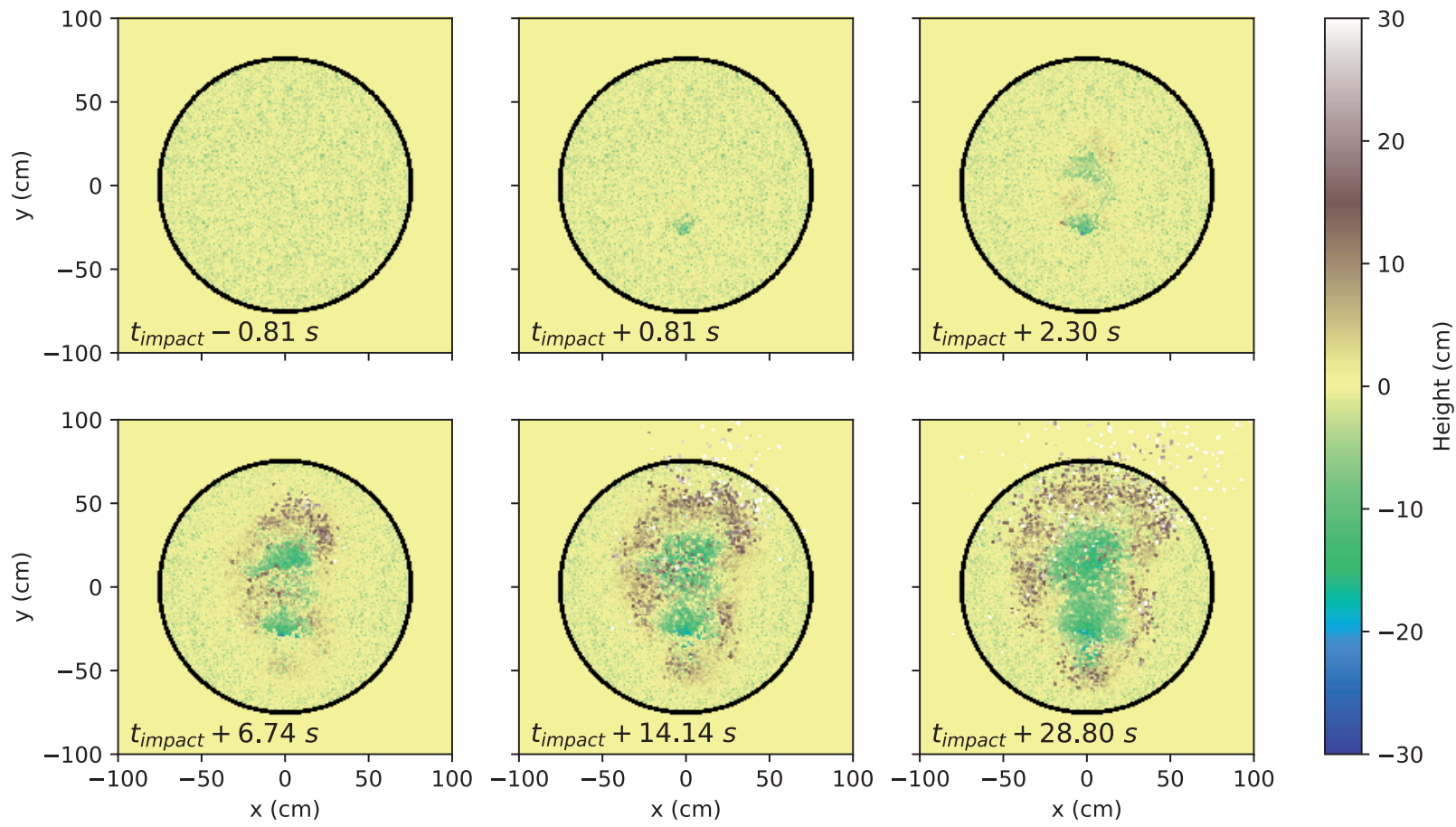

Fig. 11. Characteristics of the trace left by MASCOT on the regolith (in $\mathrm{cm}$ ) at different times for a gravel-like regolith, where $t_{\text {impact }}$ corresponds to the simulation time when MASCOT is for the first time in contact with the regolith. The color bar represents the height of the regolith (bed and ejecta). MASCOT lands in the Back-Corner-First configuration with an impact angle of $45^{\circ}$ and has no spin before the impact, and the regolith is $30 \mathrm{~cm}$ deep.
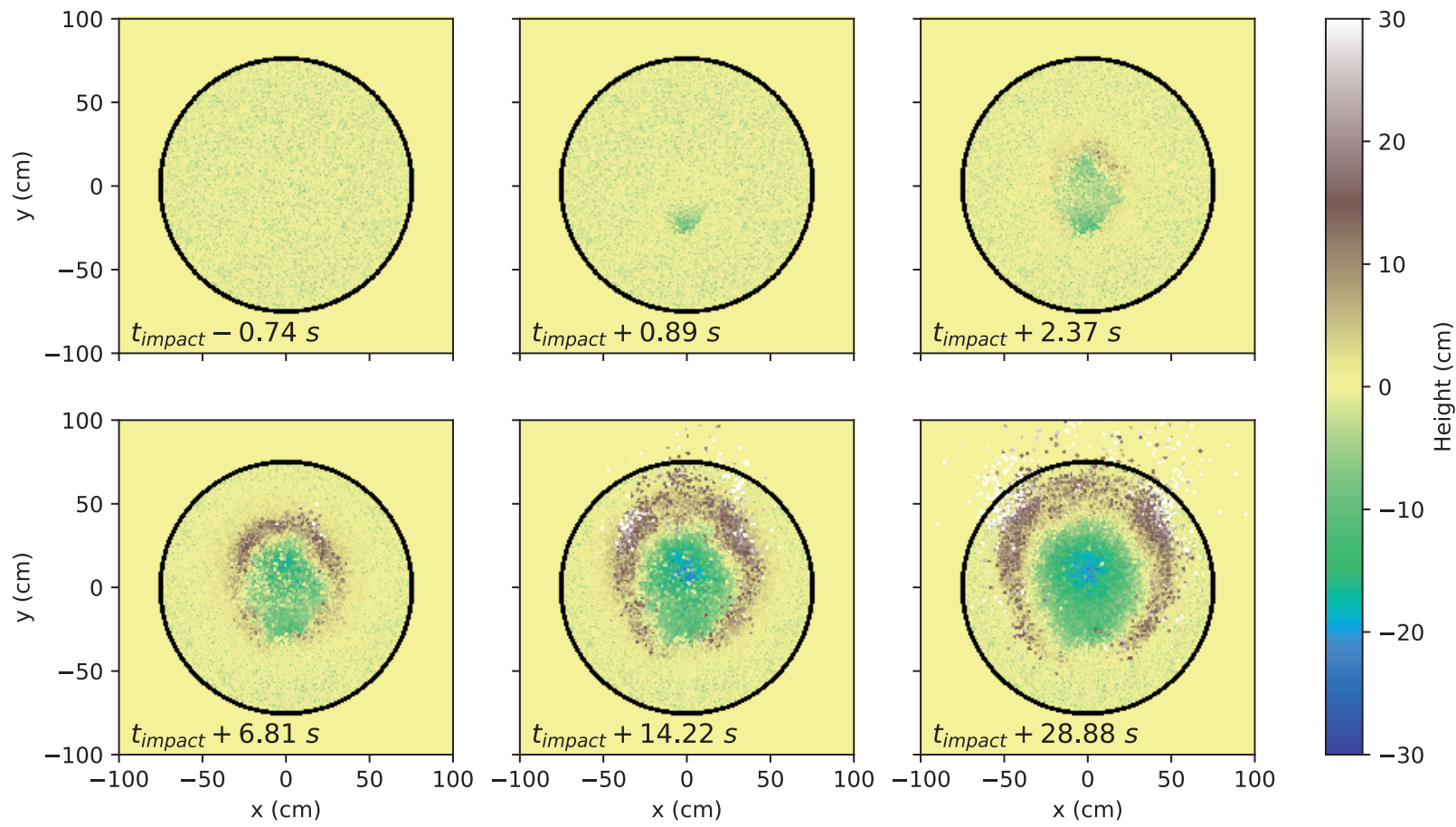

Fig. 12. Characteristics of the trace left by MASCOT on the regolith (in $\mathrm{cm}$ ) at different times for a moderate-friction regolith, where $t_{\text {impact }}$ corresponds to the simulation time when MASCOT is for the first time in contact with the regolith. The color bar represents the height of the regolith (bed and ejecta). MASCOT lands in the Back-Corner-First configuration with an impact angle of $45^{\circ}$ and has no spin before the impact, and the regolith is $30 \mathrm{~cm}$ deep. 
F. Thuillet et al.: Numerical modeling of lander interaction with a low-gravity asteroid regolith surface

Table 4. Average impact characteristics for 90 simulations with five different angles of approach, two material types, and three orientations at impact for each of the three depths considered in our simulations.

\begin{tabular}{|c|c|c|c|c|c|c|c|c|c|}
\hline Depth & $\begin{array}{l}\text { Traveled } \\
\text { distance }\end{array}$ & $\begin{array}{c}\text { Time } \\
\text { between } \\
\text { impacts }\end{array}$ & $\frac{V_{\text {out }}}{V_{\text {in }}}$ & $\frac{E_{\mathrm{rot}, \mathrm{out}}}{E_{\mathrm{lin}, \mathrm{in}}}$ & $\frac{E_{\text {rot,out }}}{E_{\text {lin,out }}}$ & $\begin{array}{l}\text { Collision } \\
\text { duration }\end{array}$ & $\begin{array}{c}\text { Maximum } \\
\text { penetration } \\
\text { depth }\end{array}$ & $\begin{array}{c}\text { Incoming angle } \\
\text { before } \\
\text { second impact }\end{array}$ & $\begin{array}{l}\text { Speed before } \\
\text { second impact }\end{array}$ \\
\hline $15 \mathrm{~cm}$ & $12.2 \mathrm{~m}$ & $252 \mathrm{~s}$ & $27 \%$ & $4.6 \%$ & $111 \%$ & 5.5 & $4.5 \mathrm{~cm}$ & $53^{\circ}$ & $5.0 \mathrm{~cm} \mathrm{~s}^{-1}$ \\
\hline $30 \mathrm{~cm}$ & $6.2 \mathrm{~m}$ & $136 \mathrm{~s}$ & $18 \%$ & $3.6 \%$ & $135 \%$ & $9.1 \mathrm{~s}$ & $8.5 \mathrm{~cm}$ & $68^{\circ}$ & $3.2 \mathrm{~cm} \mathrm{~s}^{-1}$ \\
\hline $40 \mathrm{~cm}$ & $5.4 \mathrm{~m}$ & $122 \mathrm{~s}$ & $16 \%$ & $2.9 \%$ & $149 \%$ & $10.0 \mathrm{~s}$ & $9.2 \mathrm{~cm}$ & $69^{\circ}$ & $2.7 \mathrm{~cm} \mathrm{~s}^{-1}$ \\
\hline
\end{tabular}

Notes. $V, E_{r o t}$, and $E_{\text {lin }}$ correspond respectively to MASCOT's speed, rotational energy and linear energy, and out and in to outgoing and incoming values.

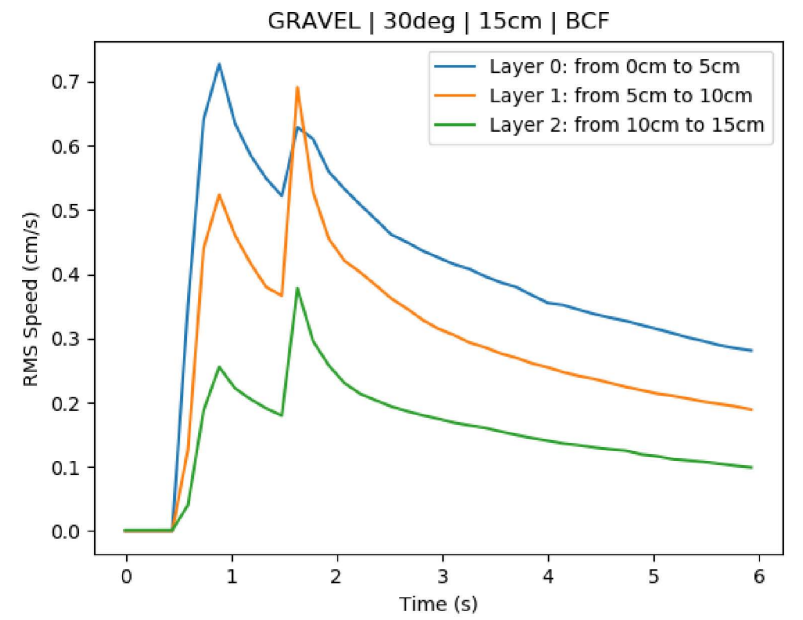

(a) $15 \mathrm{~cm}$ deep

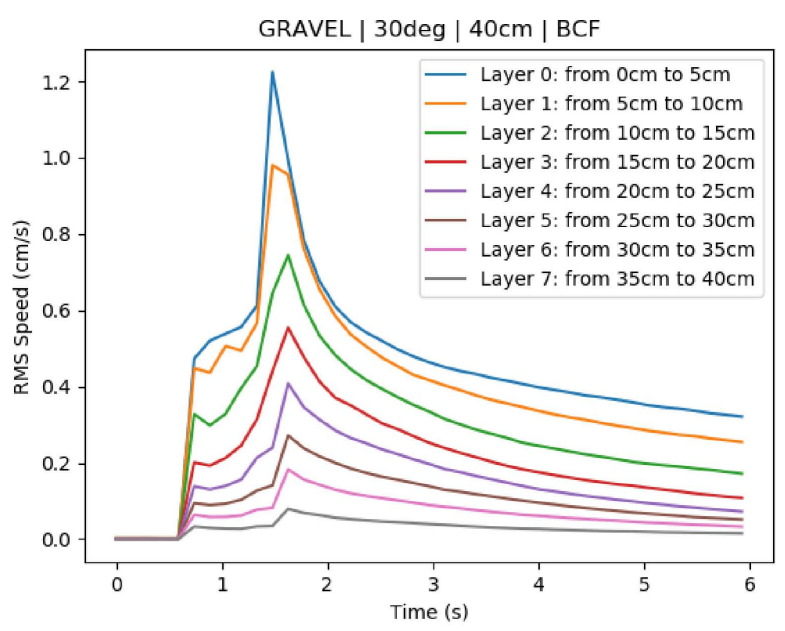

(b) $40 \mathrm{~cm}$ deep

Fig. 13. Root-mean-square (RMS) speed of the particles located in different $5 \mathrm{~cm}$ layers of the cylinder as a function of time. MASCOT lands in the Back-Corner-First configuration with an impact angle of $30^{\circ}$ on a gravel-like regolith. The bed is $15 \mathrm{~cm}$ deep in the left figure and $40 \mathrm{~cm}$ deep in the right figure.
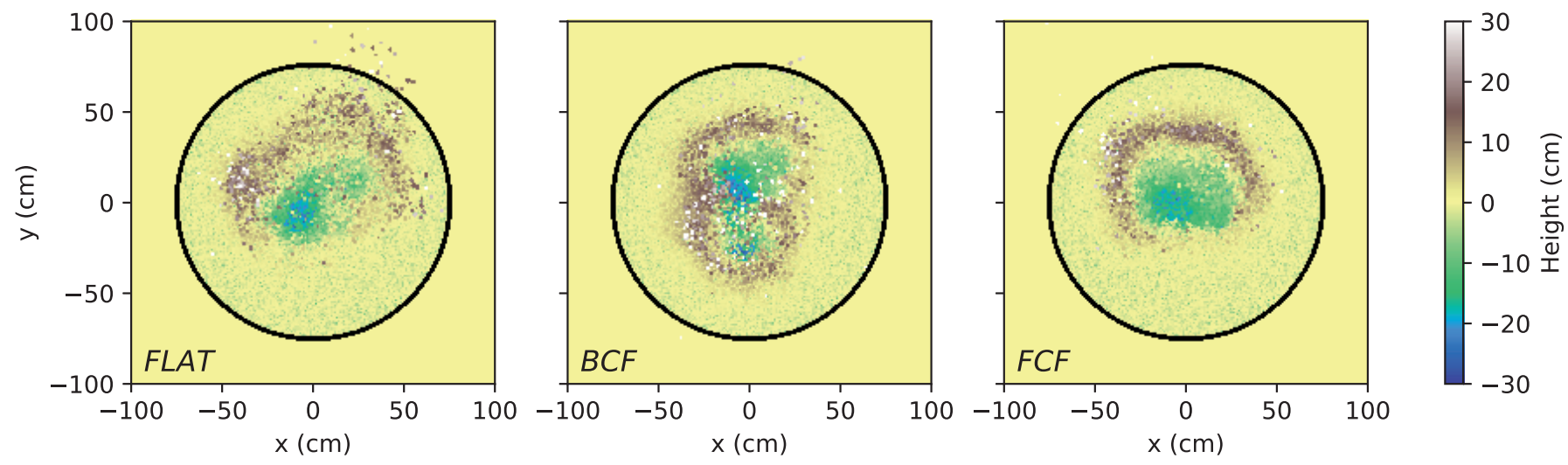

Fig. 14. Characteristics of the trace left by MASCOT on the regolith about $14 \mathrm{~s}$ after the impact for a gravel-like regolith. MASCOT lands with an impact angle of $30^{\circ}$ and the regolith is $30 \mathrm{~cm}$ deep. For the BCF impact, MASCOT left the surface after $3 \mathrm{~s}$, and after $6 \mathrm{~s}$ for the Flat impact, whereas it still has not left the surface for the FCF impact.

Figures 14 and 15 show the differences of traces depending on the orientation when MASCOT impacts the regolith bed for the two considered frictions considered. We find that the crater is shallower when MASCOT lands flat on the ground.

For a gravel-like friction (see Fig. 14), the three different orientations are discernible. In the case of MASCOT landing flat, we find a main hole and a secondary, shallower, hole. If the lander is close to horizontal, after the first corner hits the bed a second corner will impact the ground, but at a lower speed than the BCF orientation would generate, producing a second shallower hole. Since MASCOT's tangential velocity is not necessarily aligned with the first two impacting corners, the ejecta dispersion is not uniformly distributed around the two holes, and depends strongly on the corners, giving us information on the direction of MASCOT (mostly from the first hole to the second one). When the lander's orientation is no longer close to horizontal, we have either one clear deep hole (Front-Corner-First) 

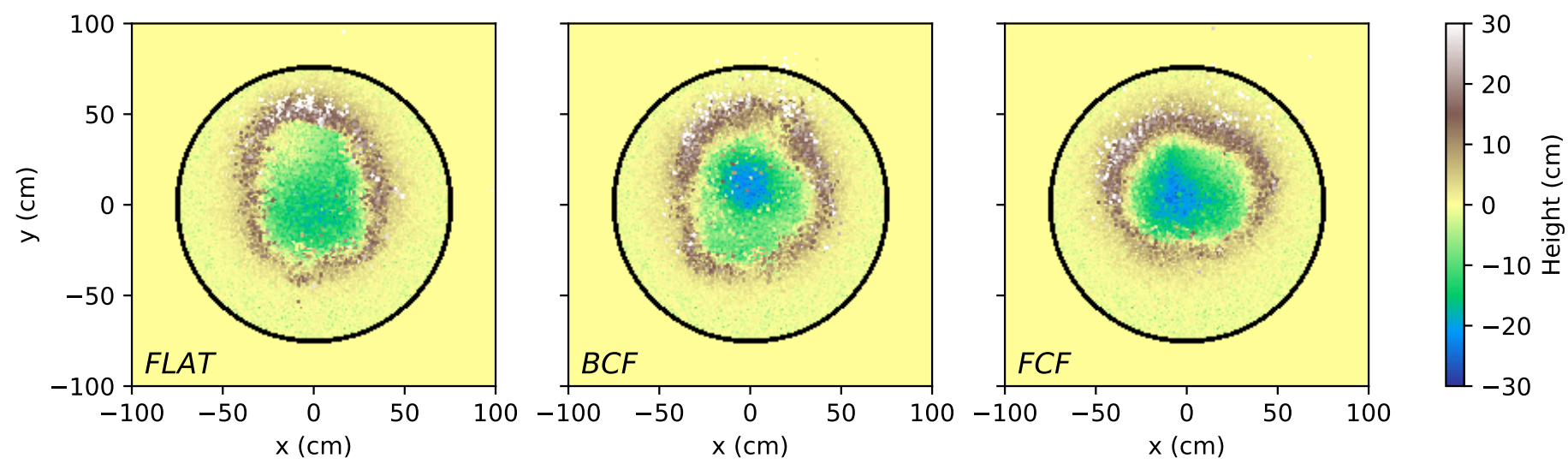

Fig. 15. Characteristics of the trace left by MASCOT on the regolith about $14 \mathrm{~s}$ after the impact for a moderate-friction regolith. MASCOT lands with an impact angle of $30^{\circ}$ and the regolith is $30 \mathrm{~cm}$ deep. For the BCF impact, MASCOT left the surface after $11.5 \mathrm{~s}$, whereas it still has not left the surface for the Flat and FCF impacts.

or two deep holes (Back-Corner-First). This process has already been described in Sect. 3.2.

With a moderate friction, the process is similar; however, since the regolith bed shows less resistance to the lander's penetration, MASCOT's bottom side also digs into the ground, leading to larger and more homogeneous traces. Moreover, whatever the orientation, the ejecta quantity is larger and goes in all directions, even if a slightly larger volume can be found in the direction MASCOT comes from. The direction MASCOT takes after the impact is within approximately ten degrees of the incoming direction. When MASCOT lands flat, it will leave on the ground a shallow and large hole. There will be a deep hole for both $\mathrm{BCF}$ and FCF orientations, but the traces still show different features. When MASCOT's front corner hits the ground first, it is barely stopped by the smooth regolith bed, and a large volume of ejecta is expelled, mostly in an ellipse whose semimajor axis is perpendicular to the direction of MASCOT. On the other hand, when the back corner first hits the bed, the bottom side drags particles, as explained in Sect. 3.2. Since the particles it hits and drags the most while pivoting are in the direction of the lander, the ellipsoidal ejecta ring will have a semimajor axis parallel to MASCOT's direction. By looking at the shape of the crater after the impact and the ejecta deposits, we can therefore deduce the orientation of MASCOT when it impacted and have a hint of the distance MASCOT travels after the first impact before impacting again.

Figure 16 confirms that the distance traveled is greatest when MASCOT lands on its back corner. Moreover, we find that the Flat configuration leads to less ejecta than the two other configurations. Indeed, when the lander hits the ground on one of its corners, it penetrates deeper into the bed and ejects a lot of particles. However, this trend is less visible for gravel-like regolith, and there is also no trend for a $15 \mathrm{~cm}$ deep bed with gravel-like friction. The post-impact spin is also higher on average when MASCOT lands on its back corner (Fig. 17). Since both speed and spin are mostly the highest with BCF, the total CoR is also the largest with this orientation.

\subsection{Maximum values}

To record the most extreme scenarios, we collected the maximum values found for each output quantity studied from all our simulations (Table 5). The maximum distances traveled (and therefore time of travel) are obtained for a $15 \mathrm{~cm}$ gravel-like bed, with an impact angle of $60^{\circ}$ from the vertical, and for MASCOT

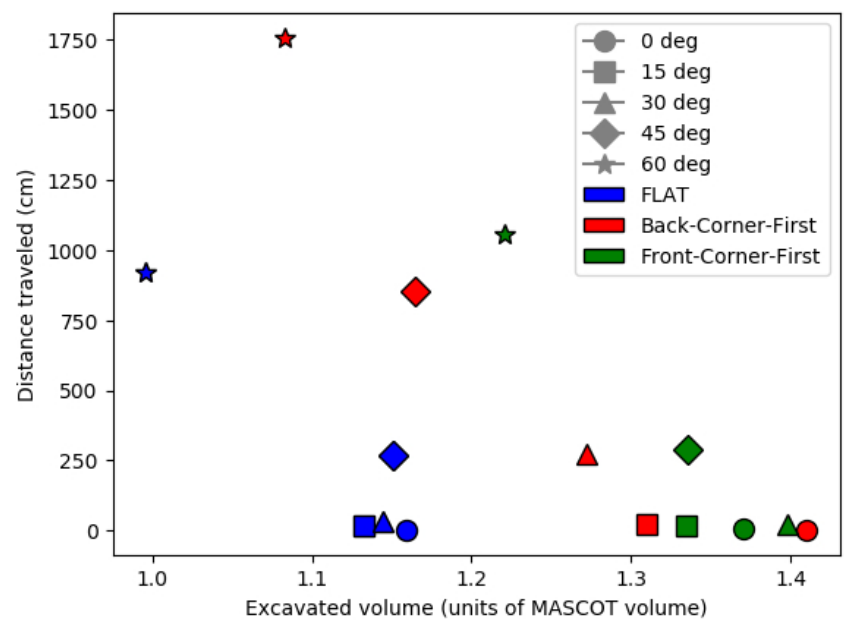

Fig. 16. Excavated volume vs. distance traveled by the lander between the first bounce and the second impact at the end of the simulation as functions of the orientation and the impact angle. MASCOT lands on a moderate-friction bed $40 \mathrm{~cm}$ in depth.

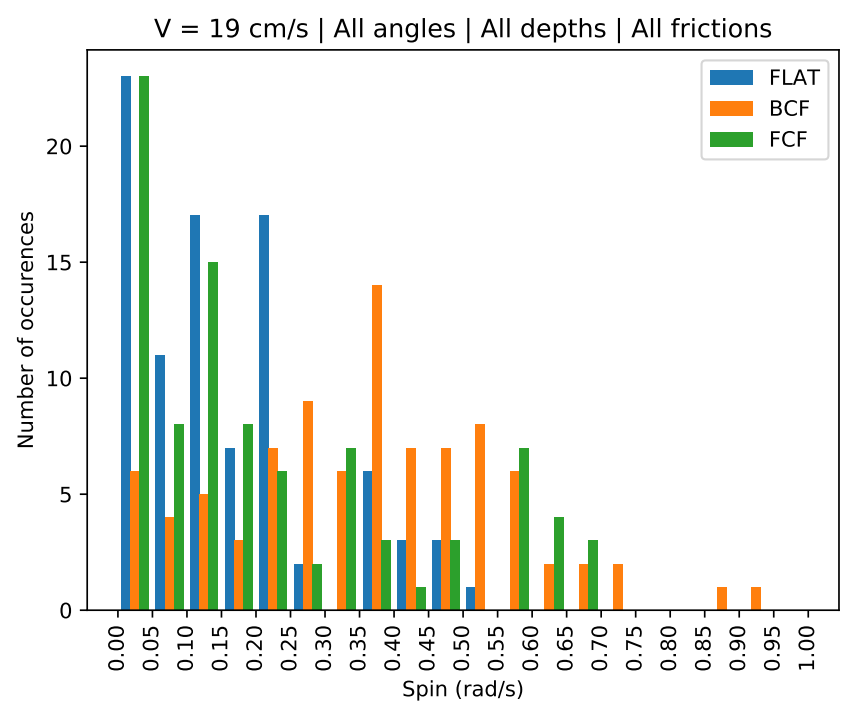

Fig. 17. Histogram of the second impact angle in the event of a second contact for the three orientations considered in this article. The data represent the five angles of impact, the three bed depths, the three orientations, and the two friction properties (total of 270 simulations). 
F. Thuillet et al.: Numerical modeling of lander interaction with a low-gravity asteroid regolith surface

Table 5. Maximum values of different impact characteristics and initial conditions of the corresponding simulations.

\begin{tabular}{cccccc}
\hline \hline \multirow{2}{*}{ Characteristic } & \multirow{2}{*}{ Value } & \multicolumn{4}{c}{ Corresponding simulation } \\
\cline { 3 - 6 } & & Friction & Angle of impact & Bed depth & Orientation \\
\hline Distance between first and second impacts & $7800 \mathrm{~cm}$ & Gravel & $60^{\circ}$ & $15 \mathrm{~cm}$ & Back-Corner-First \\
Time between first and second impacts & $810 \mathrm{~s}$ & Gravel & $60^{\circ}$ & $15 \mathrm{~cm}$ & Back-Corner-First \\
Speed before second impact & $14 \mathrm{~cm} \mathrm{~s}^{-1}$ & Gravel & $60^{\circ}$ & $15 \mathrm{~cm}$ & Back-Corner-First \\
Maximum penetration & $20.2 \mathrm{~cm}$ & Moderate & $30^{\circ}$ & $30 \mathrm{~cm}$ & Front-Corner-First \\
\hline
\end{tabular}

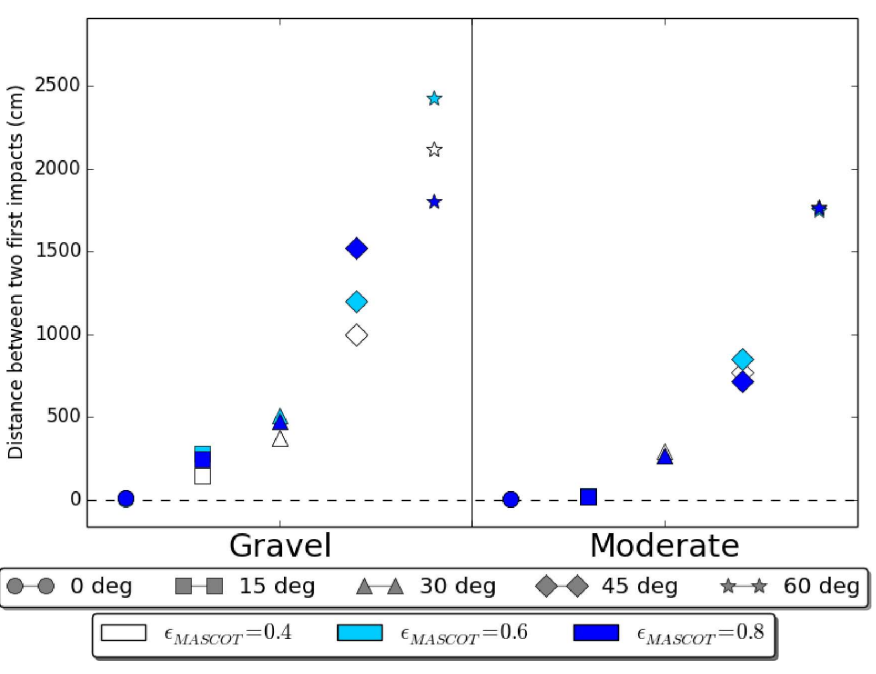

Fig. 18. Distance traveled by the lander between the first bounce and the second impact as a function of the impact angle (different marker shapes), the material type (as indicated on the plot), and the structural CoR of MASCOT (different colors). MASCOT lands in the BackCorner-First configuration with no spin before the impact, and the regolith is $40 \mathrm{~cm}$ deep.

landing on its back corner. After its first contact with the ground, the lander can travel as far as $78 \mathrm{~m}$ from the impact point, over an interval of more than 13 minutes. The corresponding simulation confirms the trends we established previously. For such distances, the curvature of the asteroid can play a role, as can a non-uniform gravity field, but we did not take these effects into account because most of our results give smaller distances where curvature and non-uniform gravity play reduced roles. Moreover, the shape of the asteroid, its gravity field, and the surface topography are still very poorly known.

The speed before the second impact can reach $14 \mathrm{~cm} \mathrm{~s}^{-1}$, from which we can expect a significant second bounce. Finding the lander may then become a challenge. However, we have seen that by looking at the crater's characteristics and the amount of ejecta during the tens of seconds following the impact, we can derive the value of the distance traveled by MASCOT within an order of magnitude and roughly guess the direction the lander took.

\subsection{Influence of MASCOT's structural coefficients of restitution}

We now analyze the influence of the coefficients of restitution, two parameters characterizing the energy dissipation. We studied separately the influence of MASCOT's coefficients of restitution and of those of the particles constituting the regolith bed.

MASCOT's normal and tangential structural CoR, due to damping inside the structure, have been measured by Biele et al.

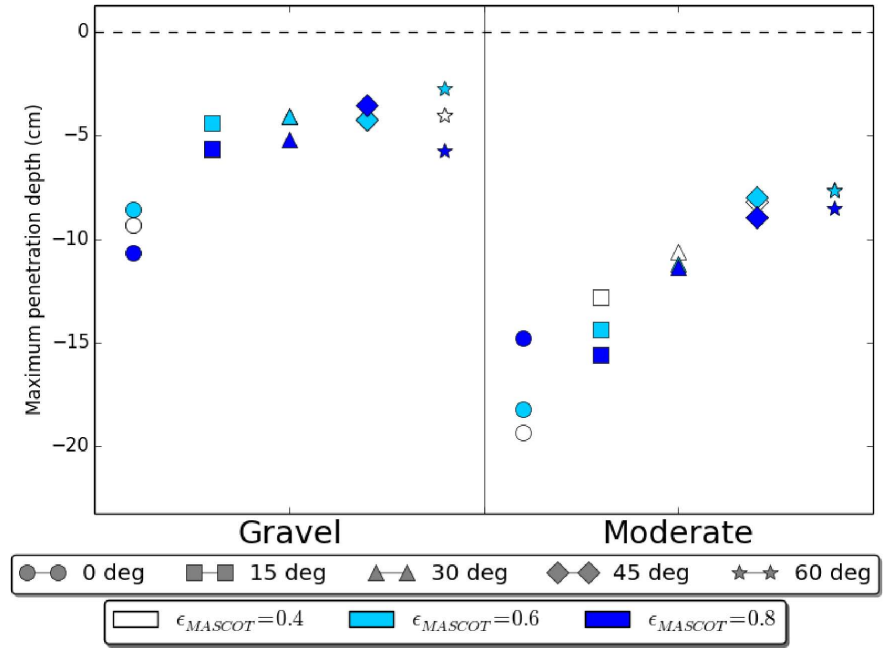

Fig. 19. Maximum penetration depth of the lander into the bed as a function of the impact angle (different marker shapes), the material type (as indicated on the plot), and the structural CoR of MASCOT (different colors). MASCOT lands in the Back-Corner-First configuration with no spin before the impact, and the regolith is $40 \mathrm{~cm}$ deep.

(2017) to be about 0.6. Since there is some uncertainty on these values, we ran a set of simulations with different values for the two structural CoRs and analyzed the results to check whether a small change in these values has a non-negligible effect on MASCOT's attitude during and after the impact. The significance of these CoRs was investigated for two different initial orientations (Flat and Back-Corner-First), and the three different bed depths $(15,30$, and $40 \mathrm{~cm})$, in addition to the usual five different angles of impact and two regolith friction values. The different values of structural CoRs considered here are 0.4, 0.6, and 0.8 , with the same value for both the normal and tangential CoRs.

As shown in Fig. 18 (distance traveled), the results may differ slightly depending on MASCOT's CoRs. However, the differences are small, and the physics of the impact and the way MASCOT interacts with the particles do not change; we therefore consider for most of the simulations CoR values of 0.6. The slight disparities of values in the three configurations confirm the nature of the bounce of MASCOT, i.e., the pivot due to MASCOT's tangential velocity before the impact. The CoR of the corner that first impacts the soil has no major influence on the dynamics of the impact, and thus on the value of the traveled distance and on its evolution as a function of the angle of impact and of the regolith friction properties.

Figure 19 gives the maximum penetration depth for the three different structural CoRs. We can see that MASCOT's different structural CoRs do not yield significant variations, meaning that even if the structural CoR were measured with slight errors, this 

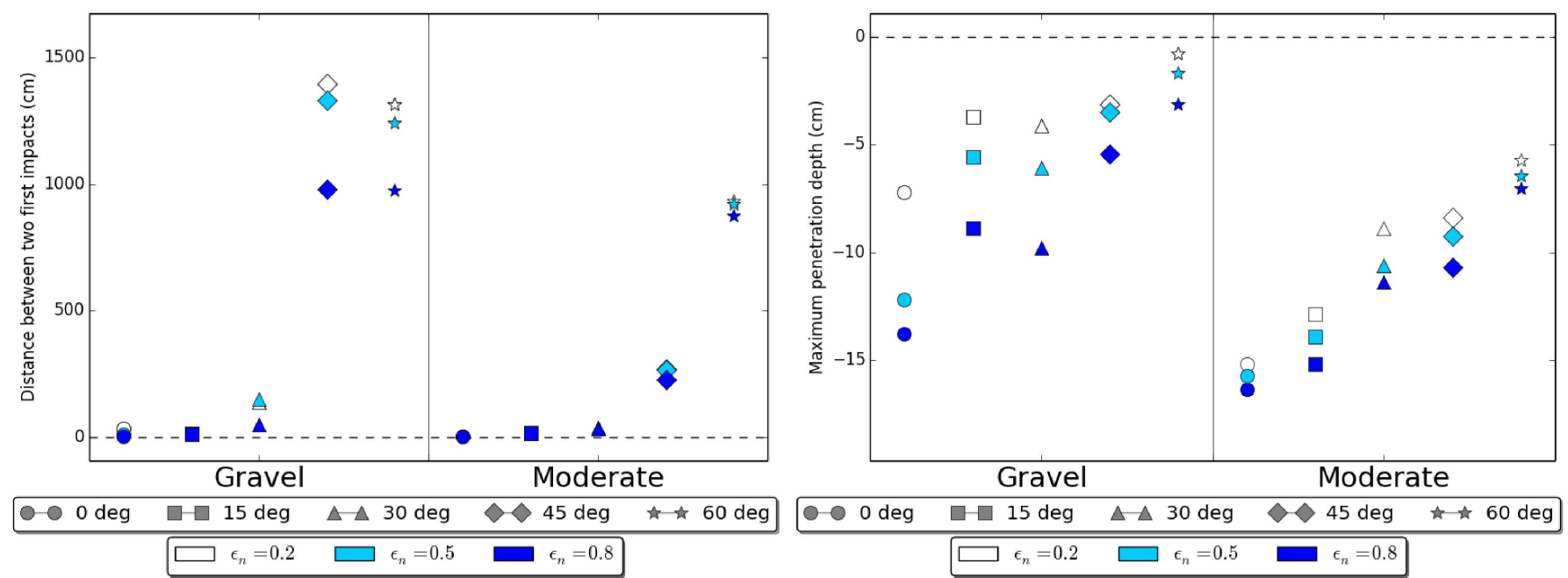

Fig. 20. Distance traveled by the lander between the first bounce and the second impact (left plot) and its maximum penetration depth into the bed (right plot) as a function of the impact angle (different marker shapes), the material type (indicated on the plots), and the normal CoR of the particles (different colors), with the tangential CoR equal to 0.5. MASCOT lands in the Flat configuration with no spin before the impact, and the regolith is $40 \mathrm{~cm}$ deep.
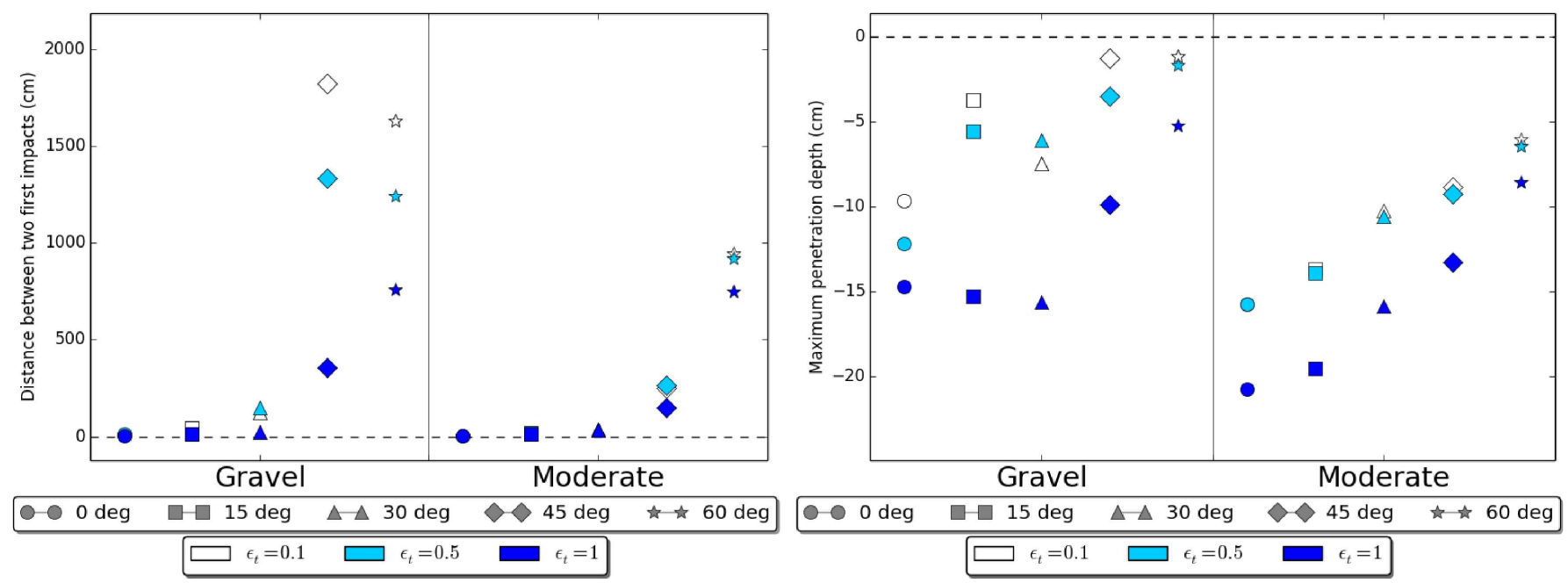

Fig. 21. Distance traveled by the lander between the first bounce and the second impact (left plot) and its maximum penetration depth into the bed (right plot) as a function of the impact angle (different marker shapes), the material type (indicated on the plots), and the tangential CoR of the particles (different colors), with the normal CoR equal to 0.5. MASCOT lands in the Flat configuration with no spin before the impact, and the regolith is $40 \mathrm{~cm}$ deep.

should not invalidate our predictions or strongly affect the values we obtained. The fact that we do not have a clear increase or decrease as a function of the structural CoR may be due to the complexity of the impact or to the stochasticity mentioned later.

\subsection{Influence of the regolith grains' coefficients of restitution}

Finally, we analyzed how the particles' CoR values affect the behavior of MASCOT during and after the impact, and how they affect the volume of particles ejected by the impact. For most of the simulations shown here we adopted $\varepsilon_{n}=\varepsilon_{t}=0.5$ (see Table 1), which is a typical choice given the angle of repose of the material considered (Maurel et al. 2017). In order to make sure that the previously established trends do not depend on specific values of particle CoRs, we ran a set of simulations with the normal $\mathrm{CoR} \varepsilon_{n}$ varying from 0.2 to 0.8 and the tangential CoR $\varepsilon_{t}$ from 0.1 to 1 .

Figure 20 shows the distance traveled by MASCOT after the first impact and the maximum penetration depth for a tangential coefficient of restitution $\varepsilon_{t}=0.5$ and different normal coefficients of restitution $\varepsilon_{n}$. A noticeable trend is that the higher the normal coefficient of restitution (for a given tangential coefficient of restitution of 0.5 ), the shorter the distance traveled and the deeper the penetration of the lander into the soil. Similarly, as shown in Fig. 21 with the same quantities but with a fixed normal CoR and different tangential CoR, the higher the coefficient, the shorter the distance and the deeper the penetration. Evidently, low coefficients of restitution for the particles make them conduct less energy between each other, and therefore the particles' speed initiated by the impact is damped in shallower layers of the regolith bed. Therefore, the bed behaves like a more rigid material, making MASCOT bounce farther, confirming the results of the simulations.

Figure 22 shows the different cases combined together for easier comparison. The plots show the results as a function of the normal CoR in the left figure and of the tangential CoR in the right one. These figures show that the higher the particles' tangential CoR, the shorter the distance traveled and the 
F. Thuillet et al.: Numerical modeling of lander interaction with a low-gravity asteroid regolith surface
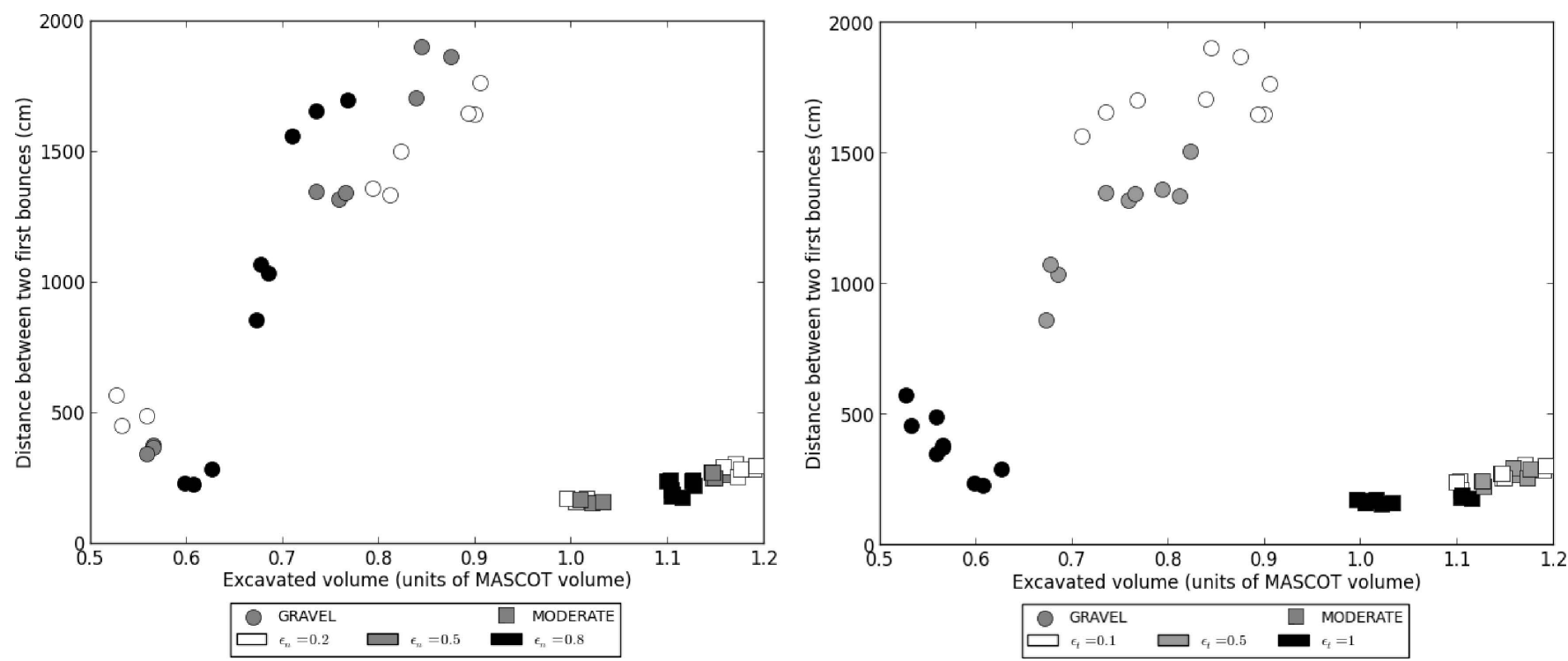

Fig. 22. Excavated volume vs. distance traveled by the lander between the first bounce and the second impact at the end of the simulation for 54 simulations for which MASCOT lands in the Flat configuration with an impact angle of $45^{\circ}$ and no spin before the impact, and the regolith is $40 \mathrm{~cm}$ deep, for various values of normal CoR $(0.2,0.5$, and 0.8$)$ and tangential CoR $(0.1,0.5$, and 1$)$. The shade of gray denotes the value of the normal CoR (left) or of the tangential CoR (right) and the symbol shape denotes the material type.

smaller the excavated volume, as mentioned previously, and we can now see that this trend does not depend on the value of the normal CoR. For the latter, although we have the same trend for the traveled distance (a decrease with increasing coefficient), the volume of ejecta seems to depend on the value of the tangential CoR. Indeed, the excavated volume seems to decrease when the normal CoR increases for low tangential CoR, but increases for high tangential CoR. Thus, further investigations are needed to establish a clearer dependence on the normal CoR.

\subsection{Stochasticity}

The behavior of MASCOT is strongly influenced by the relative position of the protuberant MicrOmega sensors (for the Flat orientation) and of the impacting corner (for the two Corner-First orientations) with respect to the grains. For example, the outcome will be different if the sensors hit the top of a grain or if they hit the surface between two grains.

Depending on the number of CPU cores or their natures, we find that there is a certain level of chaos in the system, and that particles with exactly the same initial conditions can have a slightly different position at the impact point of the lander on the regolith bed. Along these lines, we ran three simulations with similar initial bed arrangements, friction coefficients, and angles of arrival, to check that the trends that we identified are not due to these stochastic effects, and then either considered the average of all the results or considered all of them individually.

The maximum differences in position of the same particles between simulations with the same initial conditions are shown in Fig. 23. These differences are due to the regolith bed being not completely at rest and to different CPU cores computing the interactions between these particles slightly differently (we used bi CPUs Intel Xeon Ivy Bridge E5-2670 v2). Even if the differences in position are very small, these differences have an influence on the impact outcomes. For example, we computed the standard deviations for the traveled distance for three simulations with similar initial conditions (Fig. 24). For most of the simulations, the standard deviation is low enough to validate

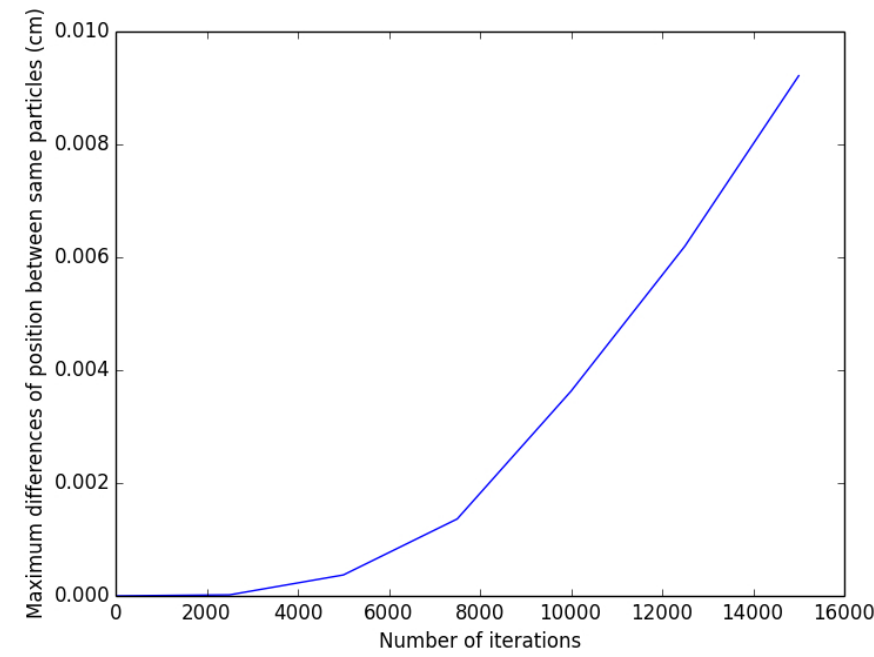

Fig. 23. Maximum differences of position for the same particles between simulations with same initial conditions as a function of time, represented by the number of iterations. These are the maxima for all the particles' differences of position of every configuration considered in this article (with $\epsilon_{n}=\epsilon_{t}=0.5$ ). The 15000 iterations correspond to about $0.44 \mathrm{~s}$ and occur just before the impact of the lander on the surface.

the trends. The standard deviation is particularly large for the simulations with a $15 \mathrm{~cm}$ bed and with grazing impacts because the traveled distances are greater. However, these larger standard deviations do not invalidate the trends described in the previous sections, as shown in Figure 2.

\section{Conclusions}

In this paper we presented our sets of numerical simulations of the low-speed impact of Hayabusa2 lander MASCOT on the surface of the asteroid Ryugu. We first investigated the influence of the depth and of two different sets of friction parameters of the regolith, as well as MASCOT's impact configuration 


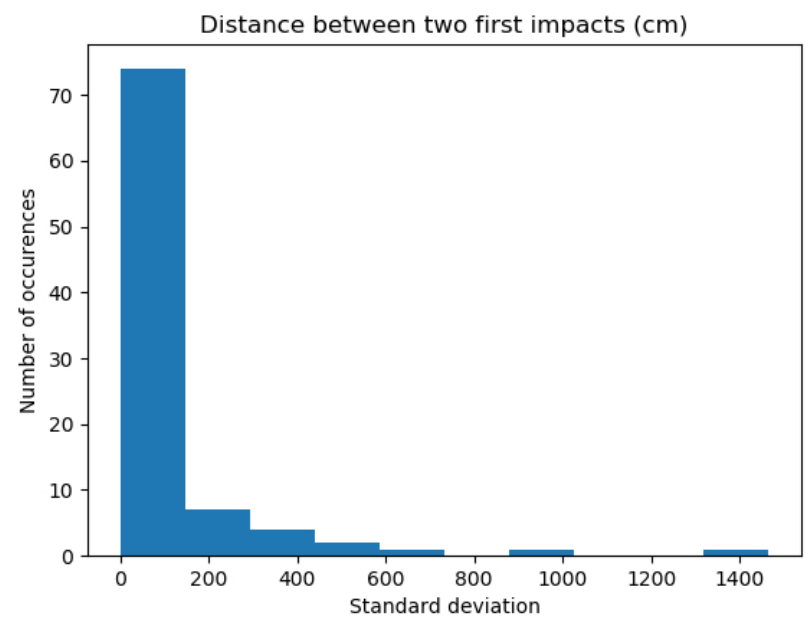

Fig. 24. Histogram of the standard deviations (in $\mathrm{cm}$ ) of the traveled distance for every configuration considered in this article (with $\epsilon_{n}=$ $\left.\epsilon_{t}=0.5\right)$.

(orientation, impact angle), on the distance traveled by the lander and the traces left on the ground.

In general, for the considered impact speed of $19 \mathrm{~cm} \mathrm{~s}^{-1}$, our simulations indicate that MASCOT is likely to bounce after its first impact. We then find that the greatest distances traveled by MASCOT after this first impact are obtained for the shallowest considered regolith bed, a gravel-like regolith, and the mostgrazing impacts of the lander. The resulting spin and speed of the lander from the first impact suggest that a second bounce cannot be ruled out, but further work is needed to determine in greater detail the evolution of the lander after the second impact. However, our results can provide standard coefficients of restitution (MASCOT's outgoing-to-incoming speed ratio, for example) and traveled distances that can serve as a reference for other software to study the whole evolution of MASCOT.

We analyzed the traces left by MASCOT on Ryugu's surface after the first impact. We find that the signature left by MASCOT is very different for the two considered types of regolith. We also find that there is a relation between the traces left by MASCOT (crater shape and ejecta deposits), and the resulting travel distance and direction after the first impact. The instruments on board Hayabusa 2 may be able to observe these impact traces, and in that case, the data will be extremely useful to give insight on both the nature of the regolith in terms of friction properties and the location of MASCOT on Ryugu's surface.

We studied the influence of the structural coefficients of restitution of MASCOT on its evolution, as these parameters are poorly constrained and may have an influence on MASCOT's behavior. The results indicate that the precise knowledge of these parameters is not essential in order to determine the processes governing the impact as they appear to have little influence on the general trends.

We looked at the influence of the two coefficients of restitutions (normal and tangential) of the regolith grains (in addition to their friction parameters). We find that the smallest values of the two coefficients of restitution result in a more rigid behavior of the regolith, and therefore result in shallower penetration into the bed as well as a greater distance traveled by the lander.
Furthermore, an increase in the tangential coefficient of restitution roughly decreases the volume of ejecta, whereas a strong trend for the normal coefficient of restitution has not been found.

The actual properties of Ryugu's regolith are not known yet, and this study considered only a limited set of possibilities that already allowed us to determine general trends for MASCOT's behavior during the first impact. This can help both engineering and scientific teams of the Hayabusa 2 mission in the search for the lander and in the determination of the regolith properties.

Further investigations will be devoted to other areas of the parameter space. In particular, the influence of the regolith packing, of a given level of cohesion between grains, and of the presence of a big boulder within the regolith or lying on it will be studied. Regarding MASCOT's impact conditions, it will also be important to consider the effect of the impact speed and other conditions within the range of possible conditions.

This study also contributes to the general understanding of the behavior of granular materials in the low-gravity environment of an asteroid's surface when experiencing an external action, here represented by the low-speed impact of a cuboid.

Acknowledgements. F.T. is supported by a PhD fellowship of the University of Nice-Sophia. P.M. and F.T. acknowledge funding support from the French space Agency CNES (BCU66), as well as from Academies of Excellence: Complex systems and Space, environment, risk, and resilience, part of the IDEX JEDI of the Université Côte d'Azur in connexion with its Center for Planetary. Simulations were performed at Mésocentre SIGAMM hosted at the Observatoire de la Côte d'Azur and on the Deepthought2 HPC cluster at the University of Maryland. E.T. and S.S. acknowledge support from the Japan Society for the Promotion of Science (JSPS) Core-to-Core program "International Network of Planetary Sciences".

\section{References}

Ballouz, R. 2017, PhD thesis, University of Maryland, College Park Biele, J., Kesseler, L., Grimm, C., et al. 2017, ArXiv e-prints, [arXiv:1705.00701]

Binzel, R. P., Harris, A. W., Bus, S. J., \& Burbine, T. H. 2001, Icarus, 151, 139

Boehnhardt, H., Bibring, J.-P., Apathy, I., et al. 2017, Phil. Trans. R. Soc. A, 375, 20160248

Delbo, M., Mueller, M., Emery, J. P., Rozitis, B., \& Capria, M. T. 2015, in Asteroids IV, eds. P. Michel, F. E. DeMeo, \& W. F. Bottke, 107

Goldman, D. I., \& Umbanhowar, P. 2008, Phys. Rev. E, 77, 021308

Gundlach, B., \& Blum, J. 2013, Icarus, 223, 479

Ho, T.-M., Baturkin, V., Grimm, C., et al. 2017, Space Sci. Rev., 208, 339

Kameda, S., Suzuki, H., Takamatsu, T., et al. 2017, Space Sci. Rev., 208, 17

Lauretta, D. S., Balram-Knutson, S. S., Beshore, E., et al. 2017, Space Sci. Rev., 212, 925

Maurel, C., Michel, P., Biele, J., Ballouz, R.-L., \& Thuillet, F. 2017, Advances in Space Research, in press, $10.1016 /$ j.asr.2017.05.029

Müller, T. G., Ďurech, J., Hasegawa, S., et al. 2011, A\&A, 525, A145

Müller, T. G., Durech, J., Ishiguro, M., et al. 2017, A\&A, 599, A103

Richardson, D., Quinn, T., Stadel, J., \& Lake, G. 2000, Icarus, 143, 45

Richardson, D., Michel, P., Walsh, K., \& Flynn, K. 2009, Planet. Space Sci., 57, 183

Richardson, D., Walsh, K., Murdoch, N., \& Michel, P. 2011, Icarus, 212, 427

Schwartz, S., Richardson, D., \& Michel, P. 2012, Granular Matter, 212, 363

Schwartz, S. R., Michel, P., Richardson, D. C., \& Yano, H. 2014, Planet. Space Sci., 103, 174

Seguin, A., Bertho, Y., \& Gondret, P. 2008, Phys. Rev. E, 78, 010301

Stadel, J. 2001, PhD thesis, University of Washington, USA

Suzuki, H., Yamada, M., Kouyama, T., et al. 2018, Icarus, 300, 341

Watanabe, S.-i., Tsuda, Y., Yoshikawa, M., et al. 2017, Space Sci. Rev., 208, 3

Yu, Y., Richardson, D. C., Michel, P., Schwartz, S. R., \& Ballouz, R.-L. 2014, Icarus, 242, 82

Zhang, Y., Richardson, D., Barnouin, O., et al. 2017, Icarus, 294, 98 